\title{
Polymorphic Subtyping for Effect Analysis: the Semantics
}

\author{
T.Amtoft \& F.Nielson \& H.R.Nielson \& J.Ammann \\ Computer Science Department, Aarhus University, Denmark \\ e-mail: \{tamtoft,fnielson, hrnielson, jammann\}@daimi.aau.dk
}

April 17, 1996

\begin{abstract}
We study an annotated type and effect system that integrates let-polymorphism, effects, and subtyping into an annotated type and effect system for a fragment of Concurrent ML. First a small step operational semantics is defined for Concurrent ML and next the annotated type and effect system is proved semantically sound. This provides insights into the rule for generalisation in the annotated type and effect system.
\end{abstract}

\section{Introduction}

In a recent paper [3] we developed an annotated type and effect system for a fragment of Concurrent ML. This system allowed the integration of ML-style polymorphism (the let-construct), subtyping (with the usual contravariant ordering for function space), and effects (for the set of "dangerous variables"). One key idea in the design of the annotated type and effect system was the following [3]:

- Carefully taking effects into account when deciding the set of variables over which to generalise in the rule for let in the inference system; this involves taking upwards closure with respect to a constraint set and is essential for maintaining semantic soundness and a number of substitution properties.

This is highlighted in the present paper. First we define a small step operational semantics [4] for Concurrent ML. It employs one system for the sequential 
components and another for the concurrent components and as in [5, 2] we use evaluation contexts [1]. Next we extend the repertoire of techniques [3] for normalising and manipulating the inference trees of the annotated type and effect system. Finally, we show that the system is indeed semantically sound with respect to the operational semantics.

\section{Inference System and Semantics}

We first briefly recapitulate the inference system presented in [3]. Expressions and constants are given by

$$
\begin{aligned}
e::= & c|x| \text { fn } x \Rightarrow e\left|e_{1} e_{2}\right| \text { let } x=e_{1} \text { in } e_{2} \\
& \mid \quad \text { rec } f x \Rightarrow e \mid \text { if } e \text { then } e_{1} \text { else } e_{2} \\
c::= & () \mid \text { true } \mid \text { false }|n|+|*|=\mid \cdots \\
& \text { pair } \mid \text { fst } \mid \text { snd } \mid \text { nil } \mid \text { cons } \mid \text { hd } \mid \text { tl } \mid \text { isnil } \\
& \text { send } \mid \text { receive } \mid \text { sync } \mid \text { channel } \mid \text { fork }
\end{aligned}
$$

where there are four kinds of constants: sequential constructors like true and pair, sequential base functions like + and $f$ st, the non-sequential constructors send and receive, and the non-sequential base functions sync, channel and fork.

Types and behaviours are given by

$$
\begin{aligned}
t & :=\alpha \mid \text { unit } \mid \text { int } \mid \text { bool }\left|t_{1} \times t_{2}\right| t \text { list } \\
& \left|t_{1} \rightarrow^{b} t_{2}\right| t \text { chan } \mid t \text { com } b \\
b & ::=\{t \text { CHAN }\}|\beta| \emptyset \mid b_{1} \cup b_{2}
\end{aligned}
$$

Type schemes ts are of form $\forall(\vec{\alpha} \vec{\beta}: C)$. $t$ with $C$ a set of constraints, where a constraint is either of form $t_{1} \subseteq t_{2}$ or of form $b_{1} \subseteq b_{2}$. The type schemes of selected constants are given in Figure 1.

Fact 2.1 Let $c$ be a constructor. Then there exists $t_{1}^{\prime}, \cdots, t_{m}^{\prime}(m \geq 0)$ and $t^{\prime}$ such that

$$
\text { TypeOf }(\mathrm{c})=\forall(\vec{\alpha} \vec{\beta}: \emptyset) \cdot t_{1}^{\prime} \rightarrow^{\emptyset} \cdots t_{m}^{\prime} \rightarrow^{\emptyset} t^{\prime}
$$

where $t^{\prime}$ is not a function type (i.e. the decomposition is "maximal") nor a type variable.

The ordering among types and behaviours is depicted in Figure 2; in particular notice that the ordering is contravariant in the argument position of a function type and that both $t$ chan $\subseteq t^{\prime}$ chan and $\{t \mathrm{CHAN}\} \subseteq\left\{t^{\prime} \mathrm{CHAN}\right\}$ demand that 


\begin{tabular}{ll}
$c$ & TypeOf $(\mathrm{c})$ \\
\hline+ & int $\times$ int $\rightarrow^{\emptyset}$ int \\
pair & $\forall\left(\alpha_{1} \alpha_{2}: \emptyset\right) \cdot \alpha_{1} \rightarrow^{\emptyset} \alpha_{2} \rightarrow^{\emptyset} \alpha_{1} \times \alpha_{2}$ \\
fst & $\forall\left(\alpha_{1} \alpha_{2}: \emptyset\right) . \alpha_{1} \times \alpha_{2} \rightarrow^{\emptyset} \alpha_{1}$ \\
snd & $\forall\left(\alpha_{1} \alpha_{2}: \emptyset\right) . \alpha_{1} \times \alpha_{2} \rightarrow^{\emptyset} \alpha_{2}$ \\
send & $\forall(\alpha: \emptyset) .(\alpha$ chan $) \times \alpha \rightarrow^{\emptyset}(\alpha$ com $\emptyset)$ \\
receive & $\forall(\alpha: \emptyset) .(\alpha$ chan $) \rightarrow^{\emptyset}(\alpha$ com $\emptyset)$ \\
sync & $\forall(\alpha \beta: \emptyset) .(\alpha \operatorname{com} \beta) \rightarrow^{\beta} \alpha$ \\
channel & $\forall(\alpha \beta:\{\{\alpha$ CHAN $\} \subseteq \beta\})$. unit $\rightarrow^{\beta} \quad(\alpha$ chan $)$ \\
fork & $\forall(\alpha \beta: \emptyset) .\left(\right.$ unit $\left.\rightarrow \rightarrow^{\beta} \alpha\right) \rightarrow^{\emptyset}$ unit
\end{tabular}

Figure 1: Type schemes for selected constants.

$t \equiv t^{\prime}$, i.e. $t \subseteq t^{\prime}$ and $t^{\prime} \subseteq t$, since $t$ occurs covariantly when used in receive and contravariantly when used in send.

The inference system is depicted in Figure 3 and employs the notion of wellformedness: a constraint set is well-formed if all constraints are of form $t \subseteq \alpha$ or $b \subseteq \beta$; and a type scheme $\forall\left(\vec{\alpha} \vec{\beta}: C_{0}\right)$. $t_{0}$ is well-formed if $C_{0}$ is well-formed and if all constraints in $C_{0}$ contain at least one variable among $\{\vec{\alpha} \vec{\beta}\}$ and if $\{\vec{\alpha} \vec{\beta}\}^{C_{0} \uparrow}=\{\vec{\alpha} \vec{\beta}\}$. Here ${ }^{1}$

$$
X^{C \uparrow}=\left\{\gamma \mid \exists \gamma^{\prime} \in X: C \vdash \gamma^{\prime} \leftarrow^{*} \gamma\right\}
$$

where the judgement $C \vdash \gamma_{1} \leftarrow \gamma_{2}$ holds if there exists $\left(g_{1} \subseteq g_{2}\right)$ in $C$ such that $\gamma_{i} \in F V\left(g_{i}\right)$ for $i=1,2$, and where we use $\leftarrow^{*}$ for the reflexive and transitive closure. Dually we have

$$
X^{C \downarrow}=\left\{\gamma \mid \exists \gamma^{\prime} \in X: C \vdash \gamma \leftarrow^{*} \gamma^{\prime}\right\}
$$

Also we write $C \vdash C_{0}$ to mean that $C \vdash g_{1} \subseteq g_{2}$ for all $g_{1} \subseteq g_{2}$ in $C_{0}$ and we say that the type scheme $\forall\left(\vec{\alpha} \vec{\beta}: C_{0}\right)$. $t_{0}$ is solvable from $C$ by $S_{0}$ if $\operatorname{Dom}\left(S_{0}\right) \subseteq\{\vec{\alpha} \vec{\beta}\}$ and if $C \vdash S_{0} C_{0}$.

\footnotetext{
${ }^{1}$ Following [3] we use $g$ to stand for $t$ or $b$ and we use $\gamma$ to stand for $\alpha$ or $\beta$ and we use $\sigma$ to stand for $t$ or $t s$.
} 
Ordering on behaviours

$$
\begin{aligned}
& \text { (axiom) } \quad C \vdash b_{1} \subseteq b_{2} \\
& \text { if }\left(b_{1} \subseteq b_{2}\right) \in C \\
& \text { (refl) } \quad C \vdash b \subseteq b \\
& \text { (trans) } \frac{C \vdash b_{1} \subseteq b_{2} \quad C \vdash b_{2} \subseteq b_{3}}{C \vdash b_{1} \subseteq b_{3}} \\
& (\mathrm{CHAN}) \quad \frac{C \vdash t \equiv t^{\prime}}{C \vdash\{t \mathrm{CHAN}\} \subseteq\left\{t^{\prime} \mathrm{CHAN}\right\}} \\
& \text { (Ø) } \quad C \vdash \emptyset \subseteq b \\
& (\cup) \quad C \vdash b_{i} \subseteq\left(b_{1} \cup b_{2}\right) \quad \text { for } i=1,2 \\
& \text { (lub) } \quad \frac{C \vdash b_{1} \subseteq b \quad C \vdash b_{2} \subseteq b}{C \vdash\left(b_{1} \cup b_{2}\right) \subseteq b}
\end{aligned}
$$

\section{Ordering on types}

$$
\begin{aligned}
& \text { (axiom) } C \vdash t_{1} \subseteq t_{2} \quad \text { if }\left(t_{1} \subseteq t_{2}\right) \in C \\
& \text { (refl) } \quad C \vdash t \subseteq t \\
& \text { (trans) } \quad \frac{C \vdash t_{1} \subseteq t_{2} \quad C \vdash t_{2} \subseteq t_{3}}{C \vdash t_{1} \subseteq t_{3}} \\
& (\rightarrow) \quad \frac{C \vdash t_{1}^{\prime} \subseteq t_{1} \quad C \vdash t_{2} \subseteq t_{2}^{\prime} \quad C \vdash b \subseteq b^{\prime}}{C \vdash\left(t_{1} \rightarrow^{b} t_{2}\right) \subseteq\left(t_{1}^{\prime} \rightarrow^{b^{\prime}} t_{2}^{\prime}\right)} \\
& (\times) \quad \frac{C \vdash t_{1} \subseteq t_{1}^{\prime} \quad C \vdash t_{2} \subseteq t_{2}^{\prime}}{C \vdash\left(t_{1} \times t_{2}\right) \subseteq\left(t_{1}^{\prime} \times t_{2}^{\prime}\right)} \\
& \text { (list) } \frac{C \vdash t \subseteq t^{\prime}}{C \vdash(t \text { list }) \subseteq\left(t^{\prime} \text { list }\right)} \\
& \text { (chan) } \frac{C \vdash t \equiv t^{\prime}}{C \vdash(t \operatorname{chan}) \subseteq\left(t^{\prime} \operatorname{chan}\right)} \\
& \text { (com) } \frac{C \vdash t \subseteq t^{\prime} \quad C \vdash b \subseteq b^{\prime}}{C \vdash(t \operatorname{com} b) \subseteq\left(t^{\prime} \operatorname{com} b^{\prime}\right)}
\end{aligned}
$$

Figure 2: Subtyping and subeffecting. 
(con) $C, A \vdash c:$ TypeOf(c) \& $\emptyset$

(id) $\quad C, A \vdash x: A(x) \& \emptyset$

(abs) $\frac{C, A\left[x: t_{1}\right] \vdash e: t_{2} \& b}{C, A \vdash \mathrm{fn} x \Rightarrow e:\left(t_{1} \rightarrow^{b} t_{2}\right) \& \emptyset}$

(app) $\frac{C_{1}, A \vdash e_{1}:\left(t_{2} \rightarrow^{b} t_{1}\right) \& b_{1} \quad C_{2}, A \vdash e_{2}: t_{2} \& b_{2}}{\left(C_{1} \cup C_{2}\right), A \vdash e_{1} e_{2}: t_{1} \&\left(b_{1} \cup b_{2} \cup b\right)}$

(let) $\frac{C_{1}, A \vdash e_{1}: t s_{1} \& b_{1} \quad C_{2}, A\left[x: t s_{1}\right] \vdash e_{2}: t_{2} \& b_{2}}{\left(C_{1} \cup C_{2}\right), A \vdash \operatorname{let} x=e_{1} \text { in } e_{2}: t_{2} \&\left(b_{1} \cup b_{2}\right)}$

(rec) $\frac{C, A[f: t] \vdash \operatorname{fn} x \Rightarrow e: t \& b}{C, A \vdash \operatorname{rec} f x \Rightarrow e: t \& b}$ $\frac{C_{0}, A \vdash e_{0}: \text { bool \& } b_{0} \quad C_{1}, A \vdash e_{1}: t \& b_{1} \quad C_{2}, A \vdash e_{2}: t \& b_{2}}{\left(C_{0} \cup C_{1} \cup C_{2}\right), A \vdash \text { if } e_{0} \text { then } e_{1} \text { else } e_{2}: t \&\left(b_{0} \cup b_{1} \cup b_{2}\right)}$

(sub) $\frac{C, A \vdash e: t \& b}{C, A \vdash e: t^{\prime} \& b^{\prime}} \quad$ if $C \vdash t \subseteq t^{\prime}$ and $C \vdash b \subseteq b^{\prime}$

(ins) $\frac{C, A \vdash e: \forall\left(\vec{\alpha} \vec{\beta}: C_{0}\right) \cdot t_{0} \& b}{C, A \vdash e: S_{0} t_{0} \& b} \quad$ if $\forall\left(\vec{\alpha} \vec{\beta}: C_{0}\right) \cdot t_{0}$ is solvable from $C$ by $S_{0}$

(gen) $\frac{C \cup C_{0}, A \vdash e: t_{0} \& b}{C, A \vdash e: \forall\left(\vec{\alpha} \vec{\beta}: C_{0}\right) \cdot t_{0} \& b} \quad \begin{aligned} & \text { if } \forall\left(\vec{\alpha} \vec{\beta}: C_{0}\right) \cdot t_{0} \text { is both well-formed, } \\ & \text { solvable from } C \text {, and satisfies }\{\vec{\alpha} \vec{\beta}\} \cap\end{aligned}$ $F V(C, A, b)=\emptyset$

Figure 3: The type inference system. 


\subsection{Properties of the Inference System}

In this paper we shall use a number of technical results from [3]; to be selfcontained we repeat their statements here.

Fact 2.2 Suppose $C \cup C_{0} \vdash \gamma_{1} \leftarrow \gamma_{2}$ with $\gamma_{1} \notin F V(C)$. Then $C_{0} \vdash \gamma_{1} \leftarrow \gamma_{2}$.

Lemma 2.3 Suppose $C$ is well-formed and that $C \vdash t \subseteq t^{\prime}$.

- If $t^{\prime}=t_{1}^{\prime} \rightarrow^{b^{\prime}} t_{2}^{\prime}$ there exist $t_{1}, t_{2}$ and $b$ such that $t=t_{1} \rightarrow^{b} t_{2}$ and such that $C \vdash t_{1}^{\prime} \subseteq t_{1}, C \vdash t_{2} \subseteq t_{2}^{\prime}$ and $C \vdash b \subseteq b^{\prime}$.

- If $t^{\prime}=t_{1}^{\prime}$ com $b^{\prime}$ there exist $t_{1}$ and $b$ such that $t=t_{1} \operatorname{com} b$ and such that $C \vdash t_{1} \subseteq t_{1}^{\prime}$ and $C \vdash b \subseteq b^{\prime}$.

- If $t^{\prime}=t_{1}^{\prime} \times t_{2}^{\prime}$ there exist $t_{1}$ and $t_{2}$ such that $t=t_{1} \times t_{2}$ and such that $C \vdash t_{1} \subseteq t_{1}^{\prime}$ and $C \vdash t_{2} \subseteq t_{2}^{\prime}$.

- If $t^{\prime}=t_{1}^{\prime}$ chan there exist $t_{1}$ such that $t=t_{1}$ chan and such that $C \vdash t_{1} \subseteq t_{1}^{\prime}$ and $C \vdash t_{1}^{\prime} \subseteq t_{1}$.

- If $t^{\prime}=t_{1}^{\prime}$ list there exist $t_{1}$ such that $t=t_{1}$ list and such that $C \vdash t_{1} \subseteq t_{1}^{\prime}$.

- If $t^{\prime}=$ int (bool, unit) then $t=$ int (bool, unit).

Lemma 2.4 Suppose that $C$ is well-formed:

if $C \vdash b \subseteq b^{\prime}$ then $F V(b)^{C \downarrow} \subseteq F V\left(b^{\prime}\right)^{C \downarrow}$.

\section{Lemma 2.5 Substitution Lemma}

For all substitutions $S$ :

(a) If $C \vdash C^{\prime}$ then $S C \vdash S C^{\prime}$.

(b) If $C, A \vdash e: \sigma \& b$ then $S C, S A \vdash e: S \sigma \& S b$ (and has the same shape).

\section{Lemma 2.6 Entailment Lemma}

For all sets $C^{\prime}$ of constraints satisfying $C^{\prime} \vdash C$ :

(a) If $C \vdash C_{0}$ then $C^{\prime} \vdash C_{0}$.

(b) If $C, A \vdash e: \sigma \& b$ then $C^{\prime}, A \vdash e: \sigma \& b$ (and has the same shape). 
Fact 2.7 Let $x$ and $y$ be distinct identifiers: if $C, A_{1}\left[x: \sigma_{1}\right]\left[y: \sigma_{2}\right] A_{2} \vdash e: \sigma \& b$ then $C, A_{1}\left[y: \sigma_{2}\right]\left[x: \sigma_{1}\right] A_{2} \vdash e: \sigma \& b$ (and has the same shape).

Fact 2.8 Let $x$ be an identifier not occurring in $e$ and let $t$ be an arbitrary type. If $C, A \vdash e: \sigma \& b$ then $C, A[x: t] \vdash e: \sigma \& b$ (and has the same shape).

Recall from [3] that an inference tree is contraint-saturated whenever all occurrences of the rules (app), (let), and (if) have the same constraints in their premises. Next recall that a strongly normalised inference tree is a constraintsaturated inference tree whose structure essentially is that of the underlying expression: the rule (ins) is only allowed immediately after a (con) or (id), the rule (gen) is only allowed immediately before a let (and only in the left branch), and the rule (sub) is never allowed after a (gen) or (sub) and is required after all other rules; we refer to [3] for the precise definition.

Fact 2.9 Enforcing Constraint-Saturation

Given an inference tree for $C, A \vdash e: \sigma \& b$ there exists a constraint-saturated inference tree $C, A \vdash_{c} e: \sigma \& b$ (that has the same shape).

\section{Lemma 2.10 Enforcing Strong Normalisation}

If $A$ is well-formed and solvable from $C$ then an inference tree $C, A \vdash e: \sigma \& b$ can be transformed into one $C, A \vdash_{s} e: \sigma \& b$ that is strongly normalised.

\subsection{The Sequential Semantics}

We are now going to define a small-step semantics for the sequential part of the language. Transitions take the form $e \rightarrow e^{\prime}$ where $e$ and $e^{\prime}$ are expressions that are essentially closed: this means that they may contain free channel identifiers ch (created by previous channel allocations) but that they must not contain any free program identifiers.

We first stipulate the semantics of the sequential base functions by means of an "evaluation function" $\delta$ :

Definition 2.11 The function $\delta$ is a partial mapping from expressions into expressions: if $\delta(e)$ is defined then $e$ will have the form $c e_{1}$ with $c$ a sequential base function (but we do not claim that it is defined on all such arguments). It is defined by the following (incomplete) table: 


\begin{tabular}{lll}
$c$ & $e$ & $\delta(c e)$ \\
\hline fst & pair $e_{1} e_{2}$ & $e_{1}$ \\
snd & pair $e_{1} e_{2}$ & $e_{2}$ \\
hd & $\operatorname{cons} e_{1} e_{2}$ & $e_{1}$ \\
tl & $\operatorname{cons} e_{1} e_{2}$ & $e_{2}$ \\
isnil & nil & true \\
isnil & $\operatorname{cons} e_{1} e_{2}$ & false \\
+ & pair $n_{1} n_{2}$ & $n$ where $n=n_{1}+n_{2}$ \\
$\vdots$ & $\vdots$ &
\end{tabular}

We next introduce the notion of weakly evaluated expressions ( $w \in W E x p$ ) that are the "terminal configurations" of the sequential semantics:

Definition 2.12 An expression $w$ is a weakly evaluated expression provided that either

- $w$ is a constant $c$; or

- $w$ is a channel identifier $c h$; or

- $w$ is a function abstraction $\mathrm{fn} x \Rightarrow e$; or

- $w$ is of form $c w_{1} \cdots w_{n}$, where $n \geq 1$, where $w_{1}, \cdots, w_{n}$ are weakly evaluated expressions, and where $c$ is a constructor (sequential or nonsequential).

To formalise the call-by-value evaluation strategy we shall employ the notion of evaluation context:

Definition 2.13 Evaluation contexts $E$ take the form

$$
E::=[]|E e| w E \mid \text { let } x=E \text { in } e \mid \text { if } E \text { then } e_{1} \text { else } e_{2}
$$

Notice that $E$ is a context with exactly one hole in it, and that this hole is not inside the scope of any defining occurrence of a program identifier. We write $E[e]$ for the expression that has the hole in $E$ replaced by $e$, and similarly $E\left[E^{\prime}\right]$ for the evalution context that results by replacing the hole in $E$ with $E^{\prime}$. The following (rather obvious) fact is proved in Appendix A:

Fact $2.14\left(E_{1}\left[E_{2}\right]\right)[e]=E_{1}\left[E_{2}[e]\right]$.

Now we are ready for:

\section{Definition 2.15 Sequential Evaluation}

The sequential transition relation $\rightarrow$ is defined by 
$E[e] \rightarrow E\left[e^{\prime}\right]$ provided $e-e^{\prime}$ holds according to the following definition:

$$
\begin{array}{rll}
\text { (apply) } & (\text { fn } x \Rightarrow e) w & \rightarrow e[w / x] \\
\text { (delta) } & c w & \rightarrow e^{\prime} \text { if } e^{\prime}=\delta(c w) \\
(\text { let }) & \text { let } x=w \text { in } e & \rightarrow e[w / x] \\
(\text { rec }) & \text { rec } f x \Rightarrow e & \rightarrow(\text { fn } x \Rightarrow e)[(\operatorname{rec} f x \Rightarrow e) / f] \\
\text { (branch) } & \text { if } w \text { then } e_{1} \text { else } e_{2} & \rightarrow \begin{cases}e_{1} & \text { if } w=\text { true } \\
e_{2} & \text { if } w=\text { false }\end{cases}
\end{array}
$$

Fact 2.16 If $e \rightarrow e^{\prime}$ with $e$ essentially closed then also $e^{\prime}$ is essentially closed.

Observe that $e_{1} e_{2} \rightarrow e^{\prime}$ holds iff either (i) $e_{1} e_{2} \rightarrow e^{\prime}$, or (ii) there exists $e_{1}^{\prime}$ such that $e_{1} \rightarrow e_{1}^{\prime}$ and $e^{\prime}=e_{1}^{\prime} e_{2}$, or (iii) there exists $e_{2}^{\prime}$ such that $e_{2} \rightarrow e_{2}^{\prime}$ and $e^{\prime}=$ $e_{1} e_{2}^{\prime}$ (in which case $e_{1}$ is a weakly evaluated expression). Further observe that let $x=e_{1}$ in $e_{2} \rightarrow e^{\prime}$ holds iff either (i) let $x=e_{1}$ in $e_{2} \rightarrow e^{\prime}$, or (ii) there exists $e_{1}^{\prime}$ such that $e_{1} \rightarrow e_{1}^{\prime}$ and $e^{\prime}=$ let $x=e_{1}^{\prime}$ in $e_{2}$. Finally observe that if $e_{0}$ then $e_{1}$ else $e_{2} \rightarrow e^{\prime}$ holds iff either (i) if $e_{0}$ then $e_{1}$ else $e_{2} \rightarrow e^{\prime}$, or (ii) there exists $e_{0}^{\prime}$ such that $e_{0} \rightarrow e_{0}^{\prime}$ and $e^{\prime}=$ if $e_{0}^{\prime}$ then $e_{1}$ else $e_{2}$.

As expected we have:

Fact 2.17 If $w$ is a weakly evaluated expression then $w \nrightarrow$.

Proof It is easy to see that $w \neq$; the result then follows by an easy induction on $w$.

We shall say that an essentially closed expression $e$ is stuck if it is not weakly evaluated and yet $e f$. We shall say that a stuck expression $e$ is top-level stuck if it cannot be written on the form $e=E\left[e^{\prime}\right]$ with $E \neq[]$ and with $e^{\prime}$ stuck. It is easy to see (using Fact 2.14) that for any stuck expression $e$ there exists $E$ and top-level stuck $e^{\prime}$ such that $e=E\left[e^{\prime}\right]$.

Fact 2.18 Suppose that $e$ is essentially closed and top-level stuck; then either

- $e=c w$ with $c$ a non-sequential base function; or

- $e=c w$ with $c$ a sequential base function where $\delta(e)$ is undefined; or

- $e=c h w$ with $c h$ a channel identifier; or

- $e=$ if $w$ then $e_{1}$ else $e_{2}$ with $w \notin\{$ true,false $\}$. 
Proof We perform a case analysis on $e$. If $e$ is a constant, a channel identifier or an abstraction then $e$ is weakly evaluated and hence not stuck. If $e$ is of form rec $f x \Rightarrow e$, then $e-\cdots$ and hence $e$ is not stuck.

If $e$ is of form let $x=e_{1}$ in $e_{2}$ then $e_{1}$ is essentially closed and $e_{1} \not \rightarrow$ (as otherwise $e \rightarrow)$ but $e_{1}$ is not stuck (as $e$ is top-level stuck). Hence we conclude that $e_{1}$ is weakly evaluated, but this is a contradiction since then $e-\cdots$.

If $e$ is of form if $e_{0}$ then $e_{1}$ else $e_{2}$ then $e_{0}$ is essentially closed and $e_{0} \rightarrow$ (as otherwise $e \rightarrow$ ) but $e_{0}$ is not stuck (as $e$ is top-level stuck). Hence we conclude that $e_{0}$ is weakly evaluated; and this yields the claim since if $e_{0}=$ true or $e_{0}=$ false then $e \rightarrow \cdots$.

If $e$ is of form $e_{1} e_{2}$ we infer (using the same technique as in the above two cases) that $e_{1}$ is a weakly evaluated expression $w_{1}$ and subsequently that $e_{2}$ is a weakly evaluated expression $w_{2}$. Since $e$ is not a weakly evaluated expression it cannot be the case that $w_{1}$ is of form $c w_{1}^{\prime} \cdots w_{n}^{\prime}$ with $c$ a constructor and with $n \geq 0$; and since $e f$ it cannot be the case that $w_{1}$ is of form $\mathrm{fn} x \Rightarrow e_{1}^{\prime}$ or a sequential base function such that $\delta(e)$ is defined. This yields the claim.

From the preceding results we get:

Proposition 2.19 Suppose that $e$ is essentially closed and that $e \rightarrow^{*} e^{\prime} f$. Then either

1. $e^{\prime}$ is a weakly evaluated expression; or

2. $e^{\prime}$ is of form $E[c w]$ with $c$ a non-sequential base function; or

3. $e^{\prime}$ is either of form $E[c w]$ with $c$ a sequential base function where $\delta(c w)$ is undefined, or of form $E[c h w]$, or of form $E\left[\right.$ if $w$ then $e_{1}$ else $\left.e_{2}\right]$ with $w \notin\{$ true, false $\}$.

The configurations listed in case 3 can be thought of as error configurations, whereas in Section 2.3 we shall see that case 2 corresponds to a process that may be able to perform a concurrent action.

Fact 2.20 The rewriting relation $\rightarrow$ is deterministic.

Proof We perform induction on $e$ to show that if $e \rightarrow e^{\prime}$ and $e \rightarrow e^{\prime \prime}$ then $e^{\prime}=e^{\prime \prime}$. If $e$ is a constant, a variable or a function abstraction then $e f$ and if $e$ is of form rec $f x \Rightarrow e$ determinism is obvious.

If $e$ is of form let $x=w$ in $e_{2}$ the claim follows from $w \neq$. If $e$ is of form let $x=e_{1}$ in $e_{2}$ with $e_{1}$ not a weakly evaluated expression then $e^{\prime}$ takes the form let $x=e_{1}^{\prime}$ in $e_{2}$ where $e_{1} \rightarrow e_{1}^{\prime}$ and by the induction hypothesis this $e_{1}^{\prime}$ is unique. 
If $e$ is of form if $w$ then $e_{1}$ else $e_{2}$ the claim follows from $w \neq$. If $e$ is of form if $e_{0}$ then $e_{1}$ else $e_{2}$ with $e_{0}$ not a weakly evaluated expression then $e^{\prime}$ takes the form if $e_{0}^{\prime}$ then $e_{1}$ else $e_{2}$ where $e_{0} \rightarrow e_{0}^{\prime}$ and by the induction hypothesis this $e_{0}^{\prime}$ is unique.

We are left with the case $e=e_{1} e_{2}$. First suppose that $e_{1}$ is not weakly evaluated. Then $e f$ and we infer that $e^{\prime}$ takes the form $e_{1}^{\prime} e_{2}$ where $e_{1} \rightarrow e_{1}^{\prime}$ so by the induction hypothesis this $e_{1}^{\prime}$ is unique.

Next suppose that $e=w_{1} e_{2}$ with $e_{2}$ not weakly evaluated. Then $e f$ and as $w_{1} \neq$ we infer that $e^{\prime}$ takes the form $w_{1} e_{2}^{\prime}$ where $e_{2} \rightarrow e_{2}^{\prime}$ so by the induction hypothesis this $e_{2}^{\prime}$ is unique.

Finally assume that $e=w_{1} w_{2}$. Then $w_{1} \not$ and $w_{2} \nrightarrow$ so it must hold that $e \rightarrow e^{\prime}$. If $w_{1}$ is a function abstraction this $e^{\prime}$ is clearly unique; and if $w_{1}$ is a sequential base function uniqueness follows from the fact that $\delta$ is a function.

\subsection{The Concurrent Semantics}

Next we are going to define a small-step semantics for the concurrent part of the language. Transitions take the form $P P \stackrel{a}{\longrightarrow} P P^{\prime}$, where $P P$ as well as $P P^{\prime}$ is a process pool which is a finite mapping from process identifiers $p$ into essentially closed expressions, and where $a$ is a label describing what kind of action is taken.

\section{Definition 2.21 Concurrent Evaluation}

The concurrent transition relation $\stackrel{a}{\longrightarrow}$ is defined by:

$$
\begin{aligned}
& P P[p: e] \underset{\text { if } e \rightarrow e^{\prime}}{\stackrel{\text { seq }}{\longrightarrow}} P P\left[p: e^{\prime}\right] \\
& P P[p: E[\text { channel () }]] \quad p \underset{\text { chan }}{\longrightarrow} \operatorname{Ph} P[p: E[c h]] \\
& \text { if } c h \text { not in } P P \text { or } E \\
& P P[p: E[\text { fork } w]] \quad \underset{\text { fork }}{\longrightarrow} p^{\prime} \quad P P[p: E[()]]\left[p^{\prime}: w()\right] \\
& \text { if } p^{\prime} \notin \operatorname{Dom}(P P) \cup\{p\} \\
& \begin{array}{c}
P P\left[p_{1}: E_{1}[\operatorname{sync}(\text { send }(\text { pair } c h w))]\right] \quad \stackrel{\text { comm }}{\longrightarrow} \quad P P\left[p_{1}: E_{1}[w]\right]\left[p_{2}: E_{2}[w]\right] \\
{\left[p_{2}: E_{2}[\text { sync }(\text { receivech })]\right]}
\end{array} \\
& \text { if } p_{1} \neq p_{2}
\end{aligned}
$$




\subsection{Manipulation of Proof Trees}

In this section we present some auxiliary results which will eventually enable us to show that if there is a typing for $e$ and if $e$ gets "rewritten" into $e^{\prime}$ (sequentially or concurrently) then we can construct a typing for $e^{\prime}$.

A common pattern will be that we have some judgement $C^{\prime}, A^{\prime} \vdash E[e]: \sigma^{\prime} \& b^{\prime}$, but we want to reason about the typing of $e$ rather than that of $E[e]$. To this end we need to be precise about what it means for a judgement to occur "at the address indicated by the hole in $E$ ":

Definition 2.22 The judgement $j d g=(C, A \vdash e: \sigma \& b)$ occurs at $E$ (with depth $n$ ) in the inference tree for the judgement $j d g^{\prime}=\left(C^{\prime}, A^{\prime} \vdash e^{\prime}: \sigma^{\prime} \& b^{\prime}\right)$, provided that either

- $j d g=j d g^{\prime}$ and $E=[]$ (and $\left.n=0\right)$; or

- there exists a judgement $j d g^{\prime \prime}$ and an evaluation context $E^{\prime \prime}$ such that $j d g$ occurs at $E^{\prime \prime}$ (with depth $n-1$ ) in the inference tree for $j d g^{\prime \prime}$, and such that the last rule applied in the inference tree for $j d g^{\prime}$ is either

- (sub), (ins), or (gen), with $j d g^{\prime \prime}$ as premise and with $E=E^{\prime \prime}$; or

- (app), with $j d g^{\prime \prime}$ as leftmost premise and with $E=E^{\prime \prime} e_{2}$ where $e^{\prime}$ is of form $e_{1} e_{2} ;$ or

- (app), with $j d g^{\prime \prime}$ as rightmost premise and with $E=w_{1} E^{\prime \prime}$ where $e^{\prime}$ is of form $w_{1} e_{2}$; or

- (let), with $j d g^{\prime \prime}$ as leftmost premise and with $E=$ let $x=E^{\prime \prime}$ in $e_{2}$ where $e^{\prime}$ is of form let $x=e_{1}$ in $e_{2}$; or

- (if), with $j d g^{\prime \prime}$ as leftmost premise and with $E=$ if $E^{\prime \prime}$ then $e_{1}$ else $e_{2}$ where $e^{\prime}$ is of form if $e_{0}$ then $e_{1}$ else $e_{2}$.

This is clearly well-defined in the size of the inference tree for $j d g^{\prime}$. As expected we have the following results, the latter to be proved in Appendix A:

Fact 2.23 Suppose that $C, A \vdash e: \sigma \& b$ occurs at $E$ in the inference tree for $C^{\prime}, A^{\prime} \vdash e^{\prime}: \sigma^{\prime} \& b^{\prime}$; then $e^{\prime}=E[e]$.

Fact 2.24 Given $j d g^{\prime}=\left(C^{\prime}, A^{\prime} \vdash E[e]: \sigma^{\prime} \& b^{\prime}\right)$; then there exists (at least one) judgement $j d g$ of form $C, A \vdash e: \sigma \& b$ such that $j d g$ occurs at $E$ in the inference tree for $j d g^{\prime}$.

Some of the subsequent proofs will be by induction in the depth of a judgement in an inference tree; for this purpose the following result is convenient: 
Fact 2.25 Suppose the judgement $j d g$ occurs at $E$ with depth $n$ in the inference tree for $j d g^{\prime}$, where $n \geq 2$. Then there exists a judgement $j d g^{\prime \prime}$ and evaluation contexts $E_{1}$ and $E_{2}$ such that

$j d g$ occurs at $E_{1}$ with depth $<n$ in the inference tree for $j d g^{\prime \prime}$; and $j d g^{\prime \prime}$ occurs at $E_{2}$ with depth $<n$ in the inference tree for $j d g^{\prime}$; and $E=E_{2}\left[E_{1}\right]$.

Proof We can clearly use $j d g^{\prime \prime}$ as in Definition 2.22 .

Having set up the necessary machinery we are now ready for the first result, which states that "equivalent" expressions may be substituted for each other:

Fact 2.26 Suppose the judgement $C, A \vdash e: \sigma \& b$ occurs at $E$ in the inference tree of $C^{\prime}, A^{\prime} \vdash E[e]: \sigma^{\prime} \& b^{\prime}$. If $e_{0}$ is such that $C, A \vdash e_{0}: \sigma \& b$ then also $C^{\prime}, A^{\prime} \vdash E\left[e_{0}\right]: \sigma^{\prime} \& b^{\prime}$.

Since the hole in an evaluation context is not inside the scope of any bound identifier we have:

Fact 2.27 Suppose the judgement $C, A \vdash e: \sigma \& b$ occurs at $E$ in the inference tree of $C^{\prime}, A^{\prime} \vdash e^{\prime}: \sigma^{\prime} \& b^{\prime}$; then $A^{\prime}=A$, and if $C^{\prime}$ is well-formed also $C$ is wellformed.

The (concurrent) transition which poses the greatest danger to semantic soundness is channel allocation, due to the need for an environment update (cf. the relationship to side effects in Standard ML). In order to construct an inference tree with the new environment we must demand that the type of the new channel is "present" in the behaviour:

Lemma 2.28 Suppose the judgement $C, A \vdash e: \sigma \& b$ occurs at $E$ in the inference tree of $C^{\prime}, A \vdash E[e]: \sigma^{\prime} \& b^{\prime}$ where $C^{\prime}$ (and hence also $C$ ) is well-formed.

Let $c h$ be a channel identifier not in $E[e]$, and let $t$ be a type and $e_{0}$ an expression such that

$$
C \vdash\{t \mathrm{CHAN}\} \subseteq b \text { and } C, A[c h: t \text { chan }] \vdash e_{0}: \sigma \& b
$$

Then it also holds that

$$
C^{\prime} \vdash\{t \mathrm{CHAN}\} \subseteq b^{\prime} \text { and } C^{\prime}, A[c h: t \text { chan }] \vdash E\left[e_{0}\right]: \sigma^{\prime} \& b^{\prime} .
$$

Proof See Appendix A.

Fact 2.27 told us something about the relationship between the root of an inference tree and the interior nodes of the tree. It proves useful to know some more: 
Lemma 2.29 Suppose the judgement $C, A \vdash e: \sigma \& b$ occurs at $E$ in the constraint-saturated inference tree of $C^{\prime}, A \vdash_{c} e^{\prime}: \sigma^{\prime} \& b^{\prime}$ where $C^{\prime}$ (and hence also $C$ ) is well-formed.

Then $C^{\prime} \subseteq C$, and there exists $S$ with $\operatorname{Dom}(S) \cap F V(A)=\emptyset$ such that $C^{\prime} \vdash S C$. Proof See Appendix A.

The following lemma tells us something about the relationship between the type of an expression $c e_{1} \cdots e_{n}$, the type of $c$, and the type of each $e_{i}$ :

Lemma 2.30 Suppose that $C$ is well-formed and that

$$
C, A \vdash_{s} c e_{1} \cdots e_{n}: t \& b(n \geq 0)
$$

and that TypeOf $(c)$ is of form

$$
\forall\left(\vec{\alpha} \vec{\beta}: C_{0}\right) \cdot t_{1}^{\prime} \rightarrow_{1}^{b_{1}^{\prime}} \cdots t_{m}^{\prime} \rightarrow_{m}^{b_{m}^{\prime}} t^{\prime \prime}
$$

where we demand that if $c$ is a base function then $m \geq n$.

Then in all cases (i.e. also if $c$ is a constructor) we can write

$$
\text { TypeOf }(c)=\forall\left(\vec{\alpha} \vec{\beta}: C_{0}\right) . t_{1}^{\prime} \rightarrow_{1}^{b_{1}^{\prime}} \cdots t_{n}^{\prime} \rightarrow_{n}^{b_{n}^{\prime}} t^{\prime}
$$

and there exists $S, t_{1} \cdots t_{n}$, and $b_{1} \cdots b_{n}$, such that

$\operatorname{Dom}(S) \subseteq\{\vec{\alpha} \vec{\beta}\}$ and $C \vdash S C_{0}$ and $C \vdash S t^{\prime} \subseteq t ;$

for all $i \in\{1 \cdots n\}: C, A \vdash_{s} e_{i}: t_{i} \& b_{i}$ and $C \vdash t_{i} \subseteq S t_{i}^{\prime}$ and $C \vdash b_{i} \subseteq b$ and $C \vdash S b_{i}^{\prime} \subseteq b$.

Similarly, if TypeOf $(c)=t_{1}^{\prime} \rightarrow_{1}^{b_{1}^{\prime}} \cdots t_{m}^{\prime} \rightarrow_{m}^{b_{m}^{\prime}} t^{\prime \prime}$ in which case $\{\vec{\alpha} \vec{\beta}\}=\emptyset$ and $C_{0}=\emptyset$ (so we have $\left.S=\mathrm{Id}\right)$.

Proof See Appendix A.

The following two lemmas, both to be proved in Appendix A, show

- that we can replace variables by expressions of the same type, provided these expressions have an empty behaviour; and

- that the latter condition can always be obtained for weakly evaluated expressions.

Lemma 2.31 Suppose that $C, A\left[x: \sigma^{\prime}\right] \vdash e: \sigma \& b$ and that $C, A \vdash e^{\prime}: \sigma^{\prime} \& \emptyset$; then $C, A \vdash e\left[e^{\prime} / x\right]: \sigma \& b$.

Lemma 2.32 Suppose that $C, A \vdash_{s} w: \sigma \& b$ with $C$ well-formed; then $C, A \vdash w: \sigma \& \emptyset$ and with the same shape. 


\section{Semantic Soundness}

In this section we shall prove that the sequential as well as the concurrent transition relation "preserves types and behaviours". First an auxiliary concept:

Definition 3.1 An environment $A$ is a channel environment if $\operatorname{Dom}(A)$ is a subset of the channel identifiers and for each $c h \in \operatorname{Dom}(A)$ that $A(c h)$ takes the form $t$ chan.

We then impose that the concurrent transition relation only operates on channel environments. This is going to hold for the initial environment which is going to be empty, and we shall see that the concurrent soundness result guarantees that the assumption is maintained; thus our decision seems to be a benign one. To see that it is actually necessary to impose the condition, note that otherwise the type of the channel would be polymorphic and the sender and receiver of a transmitted value would then be allowed to disagree on its type; this is exactly where type insecurities would creep in.

\subsection{Sequential Soundness}

First we shall prove that "top-level" reduction is sound:

Lemma 3.2 Let $C$ be well-formed and let $A$ be a channel environment and suppose

$$
C, A \vdash e: \sigma \& b
$$

If $e-e^{\prime}$ then also

$$
C, A \vdash e^{\prime}: \sigma \& b
$$

Proof Due to Lemma 2.10 (which can be applied since $A$ is trivially well-formed and solvable from $C$ ) we can assume that we in fact have $C, A \vdash_{s} e: \sigma \& b$. It will clearly suffice to show the result when $\sigma$ is a type. Moreover, it will be enough if we can show the result in the case where the last application of the rule (sub) is a trivial one. We perform case analysis on the transition $\rightarrow$ :

The transition (let) has been applied: Then the situation is

$$
\frac{C, A \vdash_{s} w: t s \& b_{1} \quad C, A[x: t s] \vdash_{s} e: t \& b_{2}}{C, A \vdash \operatorname{let} x=w \text { in } e: t \& b_{1} \cup b_{2}}
$$

and using Lemma 2.32 we have 


$$
C, A \vdash w: t s \& \emptyset
$$

which by Lemma 2.31 can be combined with the second premise of the inference to yield

$$
C, A \vdash e[w / x]: t \& b_{2}
$$

and since $C \vdash b_{2} \subseteq\left(b_{1} \cup b_{2}\right)$ we can apply (sub) to get the desired result.

The transition (rec) has been applied: Then the situation is

$$
\frac{C, A[f: t] \vdash_{s} \text { fn } x \Rightarrow e: t \& b}{C, A \vdash \operatorname{rec} f x \Rightarrow e: t \& b}
$$

and using Lemma 2.32 we have

$$
C, A[f: t] \vdash \mathrm{fn} x \Rightarrow e: t \& \emptyset
$$

so by applying (rec) we get the judgement

$$
C, A \vdash \operatorname{rec} f x \Rightarrow e: t \& \emptyset
$$

which by Lemma 2.31 can be combined with the premise of the inference to yield the desired

$$
C, A \vdash(\text { fn } x \Rightarrow e)[(\operatorname{rec} f x \Rightarrow e) / f]: t \& b .
$$

The transition (branch) has been applied: Then the situation is

$$
\frac{C, A \vdash w: \text { bool \& } b_{0} \quad C, A \vdash e_{1}: t \& b_{1} \quad C, A \vdash e_{2}: t \& b_{2}}{C, A \vdash \text { if } w \text { then } e_{1} \text { else } e_{2}: t \&\left(b_{0} \cup b_{1} \cup b_{2}\right)}
$$

and the claim is immediate.

The transition (apply) has been applied: Then the situation is

$$
\frac{\frac{C, A\left[x: t_{2}^{\prime}\right] \vdash_{s} e: t^{\prime} \& b_{0}^{\prime}}{C, A \vdash_{s} \text { fn } x \Rightarrow e: t_{2} \rightarrow^{b_{0}} t \& b_{1}}(\mathrm{abs})(\mathrm{sub}) \quad C, A \vdash_{s} w: t_{2} \& b_{2}}{C, A \vdash(\mathrm{fn} x \Rightarrow e) w: t \&\left(b_{1} \cup b_{2} \cup b_{0}\right)}
$$

where $C \vdash t_{2}^{\prime} \rightarrow b_{0}^{\prime} \quad t^{\prime} \subseteq t_{2} \rightarrow^{b_{0}} \quad t$. Since $C$ is well-formed we can apply Lemma 2.3 to deduce that 


$$
C \vdash t_{2} \subseteq t_{2}^{\prime} \text { and } C \vdash b_{0}^{\prime} \subseteq b_{0} \text { and } C \vdash t^{\prime} \subseteq t
$$

By Lemma 2.32 followed by an application of (sub) we get

$$
C, A \vdash w: t_{2}^{\prime} \& \emptyset
$$

which by Lemma 2.31 can be combined with the upmost leftmost premise of the inference to yield

$$
C, A \vdash e[w / x]: t^{\prime} \& b_{0}^{\prime}
$$

and since $C \vdash t^{\prime} \subseteq t$ and $C \vdash b_{0}^{\prime} \subseteq b_{0} \subseteq b_{1} \cup b_{2} \cup b_{0}$ we can apply (sub) to get the desired result.

The transition (delta) has been applied: The claim then follows from an examination of the table defining $\delta$; below we shall list some typical cases only. In all cases we make use of Lemma 2.30 and Lemma 2.3 which can be applied since $C$ is well-formed.

$\underline{e=\text { fst }\left(\operatorname{pair} e_{1} e_{2}\right) \text { and } \delta(e)=e_{1}:}$ Then the situation is that

$$
C, A \vdash_{s} \text { fst }\left(\text { pair } e_{1} e_{2}\right): t \& b
$$

so since TypeOf(fst) $=\forall\left(\alpha_{1} \alpha_{2}: \emptyset\right) . \alpha_{1} \times \alpha_{2} \rightarrow^{\emptyset} \alpha_{1}$ Lemma 2.30 tells us that there exists $t_{0}, b_{0}$ and $S_{0}$ such that

$$
\begin{aligned}
& C, A \vdash_{s} \text { pair } e_{1} e_{2}: t_{0} \& b_{0} \text { and } \\
& C \vdash S_{0} \alpha_{1} \subseteq t \text { and } C \vdash t_{0} \subseteq S_{0}\left(\alpha_{1} \times \alpha_{2}\right) \text { and } C \vdash b_{0} \subseteq b
\end{aligned}
$$

Since TypeOf(pair) $=\forall\left(\alpha_{1} \alpha_{2}: \emptyset\right) . \alpha_{1} \rightarrow^{\emptyset} \alpha_{2} \rightarrow^{\emptyset} \alpha_{1} \times \alpha_{2}$ Lemma 2.30 tells us that there exists $t_{1}, b_{1}, t_{2}, b_{2}$ and $S$ such that

$$
\begin{aligned}
& C, A \vdash_{s} e_{1}: t_{1} \& b_{1} \text { and } C, A \vdash_{s} e_{2}: t_{2} \& b_{2} ; \\
& C \vdash t_{1} \subseteq S \alpha_{1} \text { and } C \vdash t_{2} \subseteq S \alpha_{2} \text { and } C \vdash b_{1} \subseteq b_{0} \text { and } C \vdash b_{2} \subseteq b_{0} ; \\
& C \vdash S\left(\alpha_{1} \times \alpha_{2}\right) \subseteq t_{0} .
\end{aligned}
$$

Since $C \vdash t_{1} \times t_{2} \subseteq S \alpha_{1} \times S \alpha_{2} \subseteq t_{0} \subseteq S_{0} \alpha_{1} \times S_{0} \alpha_{2}$ we by Lemma 2.3 deduce that

$$
C \vdash t_{1} \subseteq S_{0} \alpha_{1} \subseteq t
$$

and since $C \vdash b_{1} \subseteq b_{0} \subseteq b$ we from $C, A \vdash_{s} e_{1}: t_{1} \& b_{1}$ get the desired judgement

$$
C, A \vdash e_{1}: t \& b .
$$


$\underline{e=+\left(\operatorname{pair} n_{1} n_{2}\right) \text { and } \delta(e)=n \text { where } n=n_{1}+n_{2}:}$ Then the situation is that

$$
C, A \vdash_{s}+\left(\operatorname{pair} n_{1} n_{2}\right): t \& b
$$

so since we have TypeOf $(+)=$ int $\times$ int $\rightarrow^{\emptyset}$ int we can infer by Lemma 2.30 that

$$
C \vdash \text { int } \subseteq t
$$

But as $C \vdash \emptyset \subseteq b$ this is sufficient to show the desired judgement

$$
C, A \vdash n: t \& b
$$

This completes the proof.

\section{Theorem 3.3 Sequential soundness}

Let $C$ be well-formed and let $A$ be a channel environment and suppose

$$
C, A \vdash e_{1}: \sigma \& b .
$$

If $e_{1} \rightarrow e_{2}$ then also

$$
C, A \vdash e_{2}: \sigma \& b
$$

Proof There exists $E, e_{1}^{\prime}$ and $e_{2}^{\prime}$ such that

$$
e_{1}=E\left[e_{1}^{\prime}\right] \text { and } e_{2}=E\left[e_{2}^{\prime}\right] \text { and } e_{1}^{\prime} \rightarrow e_{2}^{\prime} \text {. }
$$

By Fact 2.24 there exists $C^{\prime}, A^{\prime}, \sigma^{\prime}$ and $b^{\prime}$ such that $C^{\prime}, A^{\prime} \vdash e_{1}^{\prime}: \sigma^{\prime} \& b^{\prime}$ occurs at $E$ in the inference tree of $C, A \vdash E\left[e_{1}^{\prime}\right]: \sigma \& b$. By Fact 2.27 we infer that $A^{\prime}=A$ and that $C^{\prime}$ is well-formed. This enables us to use Lemma 3.2 from which we get

$$
C^{\prime}, A^{\prime} \vdash e_{2}^{\prime}: \sigma^{\prime} \& b^{\prime}
$$

and by Fact 2.26 we get the desired judgement

$$
C, A \vdash E\left[e_{2}^{\prime}\right]: \sigma \& b
$$

This completes the proof. 
Remark. The purpose of types is to detect certain kinds of errors at analysis time rather than at execution time. To this end one usually wants a result that guarantees that "error configurations are not typeable"; here we presuppose some well-formed constraint set and some channel environment A. By Proposition 2.19 and the discussion after it, it suffices to consider each of the error-configurations listed below, and to show that it is not typeable; for this we make use of Lemma 2.3 and Lemma 2.30 .

$c h w$ with $c h$ a channel identifier: here we employ that $A(c h)$ is of form $t$ chan.

if $w$ then $e_{1}$ else $e_{2}$ with $w \notin\{$ true,false $\}$ : for this to be typable it must hold that

$w$ can be assigned the type bool.

Thus $w$ cannot be a channel identifier (as $A$ is a channel environment); $w$ cannot be a function abstraction; and an examination of the function TypeOf will reveal that $w$ cannot be a constant (apart from true,false) or of form $c w_{1} \cdots w_{n}(n \geq 1)$ with $c$ a constructor.

$c w$ with $c$ a sequential base function where $\delta(c w)$ is undefined: consider

e.g. the expression fst $w$. For this to be typeable there must exist $t_{1}$ and $t_{2}$ such that

$w$ can be assigned the type $t_{1} \times t_{2}$.

Thus $w$ cannot be a channel identifier (as $A$ is a channel environment); $w$ cannot be a function abstraction; and an examination of the function TypeOf will reveal that $w$ cannot be a constant and that $w$ cannot be of form $c w_{1} \cdots w_{n}$ with $c$ a constructor (apart from pair).

\subsection{Concurrent Soundness}

First some auxiliary results concerning the three kinds of concurrent transitions:

Lemma 3.4 Let $C$ be well-formed and suppose that

$$
C, A \vdash_{s} E[\operatorname{channel}()]: \sigma \& b
$$

Let $c h$ be a channel identifier that does not occur in $E[\operatorname{channel}()]$; then there exists $t_{0}$ such that

$$
\begin{aligned}
& C \vdash\left\{t_{0} \mathrm{CHAN}\right\} \subseteq b \text { and } \\
& C, A\left[c h: t_{0} \text { chan }\right] \vdash E[c h]: \sigma \& b .
\end{aligned}
$$


Proof The strongly normalized inference tree contains a judgement of form

$$
C^{\prime}, A \vdash_{s} \text { channel (): } t^{\prime} \& b^{\prime}
$$

where $C^{\prime}$ is well-formed (Fact 2.27). Since

$$
\text { TypeOf(channel })=\forall(\alpha \beta:\{\{\alpha \text { CHAN }\} \subseteq \beta\}) \text { unit } \rightarrow^{\beta}(\alpha \text { chan })
$$

it follows from Lemma 2.30 that there exists $S$ such that

$$
C^{\prime} \vdash\{S \alpha \mathrm{CHAN}\} \subseteq S \beta \text { and } C^{\prime} \vdash S \alpha \text { chan } \subseteq t^{\prime} \text { and } C^{\prime} \vdash S \beta \subseteq b^{\prime}
$$

Now define $t_{0}=S \alpha$, then we have

$$
C^{\prime} \vdash\left\{t_{0} \mathrm{CHAN}\right\} \subseteq b^{\prime} \text { and } C^{\prime}, A\left[c h: t_{0} \text { chan }\right] \vdash c h: t^{\prime} \& b^{\prime}
$$

so by Lemma 2.28 we arrive at the desired relations

$$
C \vdash\left\{t_{0} \mathrm{CHAN}\right\} \subseteq b \text { and } C, A\left[c h: t_{0} \text { chan }\right] \vdash E[c h]: \sigma \& b .
$$

This completes the proof.

Lemma 3.5 Let $C$ be well-formed and suppose that

$$
C, A \vdash_{s} E[\text { fork } e]: \sigma \& b
$$

Then there exists $t^{\prime \prime}, b^{\prime \prime}$ such that

(a) $C, A \vdash E[()]: \sigma \& b$;

(b) $C, A \vdash e(): t^{\prime \prime} \& b^{\prime \prime}$.

Proof The strongly normalized inference tree contains a judgement of form

$$
C^{\prime}, A \vdash_{s} \text { forke : } t^{\prime} \& b^{\prime}
$$

and by Lemma 2.29 we infer that $C^{\prime}$ is well-formed and that there exists $S^{\prime}$ with $\operatorname{Dom}\left(S^{\prime}\right) \cap F V(A)=\emptyset$ such that $C \vdash S^{\prime} C^{\prime}$.

Since TypeOf(fork $)=\forall(\alpha \beta: \emptyset)$. (unit $\left.\rightarrow^{\beta} \alpha\right) \rightarrow^{\emptyset}$ unit Lemma 2.30 tells us that there exists $t_{1}, b_{1}$ and $S$ such that 


$$
\begin{aligned}
& C^{\prime} \vdash \text { unit } \subseteq t^{\prime} \\
& C^{\prime}, A \vdash_{s} e: t_{1} \& b_{1} \text { and } C^{\prime} \vdash t_{1} \subseteq \text { unit } \rightarrow S \beta \quad S \alpha
\end{aligned}
$$

Here (1) (together with $C^{\prime} \vdash \emptyset \subseteq b^{\prime}$ ) tells us that

$$
C^{\prime}, A \vdash(): t^{\prime} \& b^{\prime}
$$

which by Fact 2.26 yields claim (a).

For claim (b) we use Lemma 2.3 on (2) to find $t_{1}^{\prime}, b_{1}^{\prime}, t_{1}^{\prime \prime}$ such that

$$
C^{\prime}, A \vdash_{s} e: t_{1}^{\prime} \rightarrow_{1}^{b_{1}^{\prime}} t_{1}^{\prime \prime} \& b_{1} \text { and } C^{\prime} \vdash \text { unit } \subseteq t_{1}^{\prime} .
$$

This shows that

$$
C^{\prime}, A \vdash e(): t_{1}^{\prime \prime} \& b_{1}^{\prime \prime} \text { for some } b_{1}^{\prime \prime}
$$

and by Lemma 2.5 and Lemma 2.6 ( since $C \vdash S^{\prime} C^{\prime}$ ) this yields the desired judgement

$$
C, A \vdash e(): S^{\prime} t_{1}^{\prime \prime} \& S^{\prime} b_{1}^{\prime \prime} \text {. }
$$

This completes the proof.

Lemma 3.6 Let $C$ be well-formed and let $A$ be a channel environment and suppose that

$$
C, A \vdash_{s} E_{1}[\operatorname{sync}(\text { send }(\text { pairch } w))]: \sigma_{1} \& b_{1}
$$

and suppose that

$$
C, A \vdash_{s} E_{2}[\operatorname{sync}(\text { receive } c h)]: \sigma_{2} \& b_{2} \text {. }
$$

Let $A(c h)=t$ chan, then

(a) $C, A \vdash E_{1}[w]: \sigma_{1} \& b_{1}$;

(b) $C, A \vdash w: t \& \emptyset$;

(c) $C, A \vdash E_{2}[w]: \sigma_{2} \& b_{2}$.

Proof The tree (3) will contain a judgement of form 


$$
\left.C_{1}, A \vdash_{s} \text { sync (send }(\operatorname{pair} c h w)\right): t_{1} \& b_{1}^{\prime}
$$

with $C_{1}$ well-formed. Since TypeOf(sync $)=\forall(\alpha \beta: \emptyset) .(\alpha \operatorname{com} \beta) \rightarrow^{\beta} \alpha$ Lemma 2.30 tells us that there exists $t_{3}, b_{3}$ and $S_{3}$ such that

$$
\begin{aligned}
& C_{1}, A \vdash_{s} \text { send }(\text { pairch } w): t_{3} \& b_{3} ; \\
& C_{1} \vdash S_{3} \alpha \subseteq t_{1} ; \\
& C_{1} \vdash t_{3} \subseteq\left(S_{3} \alpha\right) \text { com }\left(S_{3} \beta\right) ; \\
& C_{1} \vdash b_{3} \subseteq b_{1}^{\prime} .
\end{aligned}
$$

Since TypeOf(send) $=\forall(\alpha: \emptyset) . \alpha$ chan $\times \alpha \rightarrow^{\emptyset} \alpha$ com $\emptyset$ Lemma 2.30 tells us that there exists $t_{4}, b_{4}$ and $S_{4}$ such that

$$
\begin{aligned}
& C_{1}, A \vdash_{s} \text { pairchw: } t_{4} \& b_{4} ; \\
& C_{1} \vdash\left(S_{4} \alpha\right) \text { com } \emptyset \subseteq t_{3} ; \\
& C_{1} \vdash t_{4} \subseteq\left(S_{4} \alpha\right) \text { chan } \times\left(S_{4} \alpha\right) \\
& C_{1} \vdash b_{4} \subseteq b_{3} .
\end{aligned}
$$

Since TypeOf(pair) $=\forall\left(\alpha_{1} \alpha_{2}: \emptyset\right) . \alpha_{1} \rightarrow^{\emptyset} \alpha_{2} \rightarrow^{\emptyset} \alpha_{1} \times \alpha_{2}$ Lemma 2.30 tells us that there exists $t_{5}, b_{5}, t_{6}, b_{6}$ and $S_{5}$ such that

$$
\begin{aligned}
& C_{1}, A \vdash_{s} \text { ch: } t_{5} \& b_{5} ; \\
& C_{1}, A \vdash_{s} w: t_{6} \& b_{6} ; \\
& C_{1} \vdash S_{5} \alpha_{1} \times S_{5} \alpha_{2} \subseteq t_{4} ; \\
& C_{1} \vdash t_{5} \subseteq S_{5} \alpha_{1} \text { and } C_{1} \vdash t_{6} \subseteq S_{5} \alpha_{2} ; \\
& C \vdash b_{6} \subseteq b_{4} .
\end{aligned}
$$

Since $A(c h)=t$ chan we infer from (6) that

$$
C_{1} \vdash t \operatorname{chan} \subseteq t_{5}
$$

We now apply Lemma 2.3 repeatedly: from

$$
\begin{aligned}
& C_{1} \vdash\left(S_{4} \alpha\right) \text { com } \emptyset \subseteq t_{3} \subseteq\left(S_{3} \alpha\right) \text { com }\left(S_{3} \beta\right) \text { and } \\
& C_{1} \vdash t_{5} \times t_{6} \subseteq S_{5} \alpha_{1} \times S_{5} \alpha_{2} \subseteq t_{4} \subseteq\left(S_{4} \alpha\right) \text { chan } \times\left(S_{4} \alpha\right)
\end{aligned}
$$

we deduce that

$$
\begin{aligned}
& C_{1} \vdash S_{4} \alpha \subseteq S_{3} \alpha \subseteq t_{1} \text { and } \\
& C_{1} \vdash t \text { chan } \subseteq t_{5} \subseteq\left(S_{4} \alpha\right) \text { chan and } \\
& C_{1} \vdash t_{6} \subseteq S_{4} \alpha
\end{aligned}
$$


By applying Lemma 2.3 once more ${ }^{2}$, exploiting the contravariance of $\cdots$ chan (cf. the remarks concerning Figure 2), we end up with the following relations:

$$
C_{1} \vdash t_{6} \subseteq t_{1} \text { and } C_{1} \vdash t_{6} \subseteq t
$$

As $C_{1} \vdash b_{6} \subseteq b_{1}^{\prime}$ we get from (7) that

$$
C_{1}, A \vdash w: t_{1} \& b_{1}^{\prime} \text {, }
$$

which by Fact 2.26 yields claim (a); next using Lemma 2.32 on (7) we also get

$$
C_{1}, A \vdash w: t \& \emptyset \text {. }
$$

By Lemma 2.29 there exists $S_{1}$ with $\operatorname{Dom}\left(S_{1}\right) \cap F V(A)=\emptyset$ such that $C \vdash S_{1} C_{1}$, so by applying Lemma 2.5 and Lemma 2.6 we arrive at

$$
C, A \vdash w: S_{1} t \& \emptyset
$$

which yields the claim (b) since $F V(t) \subseteq F V(A)$ and hence $S_{1} t=t$.

Our remaining task is to show claim (c), where we first notice that the tree (4) will contain a judgement of form

$$
\left.C_{2}, A \vdash_{s} \text { sync (receive } c h\right): t_{2} \& b_{2}^{\prime}
$$

with $C_{2}$ well-formed. Since TypeOf(sync $)=\forall(\alpha \beta: \emptyset) .(\alpha \operatorname{com} \beta) \rightarrow^{\beta} \alpha$ Lemma 2.30 tells us that there exists $t_{7}, b_{7}$ and $S_{7}$ such that

$$
\begin{aligned}
& C_{2}, A \vdash_{s} \text { receivech }: t_{7} \& b_{7} ; \\
& C_{2} \vdash S_{7} \alpha \subseteq t_{2} ; \\
& C_{2} \vdash t_{7} \subseteq\left(S_{7} \alpha\right) \operatorname{com}\left(S_{7} \beta\right) .
\end{aligned}
$$

Since TypeOf(receive $)=\forall(\alpha: \emptyset) .(\alpha \operatorname{chan}) \rightarrow^{\emptyset}(\alpha \operatorname{com} \emptyset)$ Lemma 2.30 tells us that there exists $t_{8}, b_{8}$ and $S_{8}$ such that

$$
\begin{aligned}
& C_{2}, A \vdash_{s} \text { ch : } t_{8} \& b_{8} ; \\
& C_{2} \vdash\left(S_{8} \alpha\right) \text { com } \emptyset \subseteq t_{7} ; \\
& C_{2} \vdash t_{8} \subseteq\left(S_{8} \alpha\right) \text { chan. }
\end{aligned}
$$

Since $A(c h)=t$ chan we infer from (9) that

\footnotetext{
${ }^{2}$ For later reference we note that if we were to use also the covariance of $\cdots$ chan we would additionally get that $C_{1} \vdash t \subseteq S_{4} \alpha \subseteq t_{1}$.
} 


$$
C_{2} \vdash t \operatorname{chan} \subseteq t_{8}
$$

We now apply Lemma 2.3 repeatedly: from

$$
\begin{aligned}
& C_{2} \vdash\left(S_{8} \alpha\right) \text { com } \emptyset \subseteq t_{7} \subseteq\left(S_{7} \alpha\right) \text { com }\left(S_{7} \beta\right) \text { and } \\
& C_{2} \vdash t \text { chan } \subseteq t_{8} \subseteq\left(S_{8} \alpha\right) \text { chan }
\end{aligned}
$$

we get, by exploiting the covariance of $\cdots$ chan (cf. the remarks concerning Figure 2),

$$
C_{2} \vdash t \subseteq S_{8} \alpha \subseteq S_{7} \alpha \subseteq t_{2}
$$

Since Lemma 2.29 ensures that $C \subseteq C_{2}$ we can deduce from claim (b) that

$$
C_{2}, A \vdash w: t \& \emptyset
$$

so by applying (sub) we arrive at

$$
C_{2}, A \vdash w: t_{2} \& b_{2}^{\prime}
$$

which by Fact 2.26 yields claim (c).

We are now able to formulate what it means for our system to be semantically sound. We write $C, A \vdash P P: P T \& P B$, where $P T$ (respectively $P B$ ) is a mapping from process identifiers into types (respectively behaviours), if the domains of $P P, P T$ and $P B$ are equal and if $C, A \vdash P P(p): P T(p) \& P B(p)$ for all $p \in \operatorname{Dom}(P P)$.

Theorem 3.7 Semantic (concurrent) soundness

Let $C$ be well-formed and let $A$ be a channel environment and suppose

$$
C, A \vdash P P: P T \& P B .
$$

If $P P \stackrel{a}{\longrightarrow} P P^{\prime}$ then there exists $P T^{\prime}, P B^{\prime}$ and channel environment $A^{\prime}$ such that

$$
C, A^{\prime} \vdash P P^{\prime}: P T^{\prime} \& P B^{\prime}
$$

and such that if $p$ is in the domain of $P P$ then $P T^{\prime}(p)=P T(p)$ and $P B^{\prime}(p)=$ $P B(p)$ and such that if ch occurs in $P P$ then $A^{\prime}(c h)=A(c h)$.

Furthermore we have the following property:

- if $a=p$ chan $c h$ then there exists $t_{0}$ such that $C \vdash\left\{t_{0} \mathrm{CHAN}\right\} \subseteq P B(p)$ and such that $A^{\prime}(c h)=t_{0}$ chan.

Proof Notice that by Lemma 2.10 we can assume that the inference trees in $C, A \vdash P P: P T \& P B$ are strongly normalised. We perform case analysis on the action label $a$ : 
$a=$ seq: $\quad$ It follows from Theorem 3.3 that we can use $P T^{\prime}=P T, P B^{\prime}=P B$ and $A^{\prime}=A$.

$a=p$ chan $c h$ : It follows from Lemma 3.4 that there exists $t_{0}$ such that the claim follows with $P T^{\prime}=P T, P B^{\prime}=P B$ and $A^{\prime}=A\left[\right.$ ch: $t_{0}$ chan]. (For $p^{\prime}$ in the domain of $P P$ with $p^{\prime} \neq p$ we must show that $C, A \vdash P P\left(p^{\prime}\right): P T\left(p^{\prime}\right) \& P B\left(p^{\prime}\right)$ implies $C, A^{\prime} \vdash P P\left(p^{\prime}\right): P T\left(p^{\prime}\right) \& P B\left(p^{\prime}\right)$, but this follows from Fact 2.8.)

$a=p$ fork $p^{\prime}$ : It follows from Lemma 3.5 that there exists $t^{\prime \prime}, b^{\prime \prime}$ such that we can use $P T^{\prime}=P T\left[p^{\prime}: t^{\prime \prime}\right], P B^{\prime}=P B\left[p^{\prime}: b^{\prime \prime}\right]$ and $A^{\prime}=A$.

$\underline{a=\mathrm{comm}:}$ It follows from Lemma 3.6 that we can use $P T^{\prime}=P T, P B^{\prime}=P B$ and $A^{\prime}=A$.

Remark. Theorem 3.7 says that if we start with a correctly typed program then we are never going to encounter programs that are not correctly typed. One consequence of this is that Lemma 3.6 will be applicable at all stages; this is a result that ensures that the value sent can always be given the type allowed on the channel on which it was sent, that having sent the value we still have a correctly typed sender, and that having received the value we still have a correctly typed receiver. However, the statement of Lemma 3.6 does not directly relate:

- the type $t_{6}$ of the value $w$ actually communicated (see line 7),

- the type $t$ of the entities allowed to be communicated over the channel,

- the type $t_{1}$ that the sender thinks was communicated (see line 5 ), and

- the type $t_{2}$ that the receiver thinks was communicated (see line 8).

However, by inspecting the proof of Lemma 3.6 one may note that the following relations are established:

$$
C_{1} \vdash t_{6} \subseteq t \quad C_{1} \vdash t \subseteq t_{1} \quad C_{2} \vdash t \subseteq t_{2}
$$

Here the constraint sets $C_{1}$ and $C_{2}$ are those corresponding to the point of sending and receiving, respectively. Thus we can be ensured that a value is always received with a type that is larger than the type it actually had when communicated. (It is possible for the sender to think that an even larger type was communicated, but this causes no harm.) 


\section{Conclusion}

We have given a formal justification of the semantic soundness of a previously developed annotated type and effect system that integrates polymorphism, subtyping and effects [3]. Although the development was performed for a fragment of Concurrent ML we believe it equally possible for Standard ML with references.

Acknowledgement This work has been supported in part by the $D A R T$ project (Danish Natural Science Research Council) and the LOMAPS project (ESPRIT BRA project 8130 ); it represents joint work among the authors.

\section{References}

[1] M.Felleisen, D.P.Friedman: Control Operators, the SECD-Machine, and the $\lambda$-calculus. Formal Descriptions of Programming Concepts III, NorthHolland, 1986.

[2] H.R. Nielson and F. Nielson. Higher-order Concurrent Programs with Finite Communication Topology. In Proc. POPL'94, pages 84-97. ACM Press, 1994.

[3] H.R.Nielson, F.Nielson, T.Amtoft: Polymorphic Subtypes for Effect Analysis: the Integration, 1996.

[4] G.D.Plotkin: A Structural Approach to Operational Semantics, Report DAIMI FN-19, Aarhus University, Denmark, 1981.

[5] J. H. Reppy. Concurrent ML: Design, Application and Semantics. In Proc. Functional Programming, Concurrency, Simulation and Automated Reasoning, pages 165-198. SLNCS 693, 1993. 


\section{A Details of Proofs}

\section{The sequential semantics}

Fact $2.14\left(E_{1}\left[E_{2}\right]\right)[e]=E_{1}\left[E_{2}[e]\right]$.

Proof The proof is by induction in $E_{1}$. If $E_{1}=$ [] the equation reads $E_{2}[e]=$ $E_{2}[e]$, so assume that $E_{1}$ is a composite context and let us consider the case $E_{1}=E e_{2}$ (the other cases are similar). By using the induction hypothesis for $E$ we get the desired equation

$$
E_{1}\left[E_{2}\right][e]=\left(E e_{2}\right)\left[E_{2}\right][e]=\left(E\left[E_{2}\right] e_{2}\right)[e]=E\left[E_{2}\right][e] e_{2}=E\left[E_{2}[e]\right] e_{2}=E_{1}\left[E_{2}[e]\right] .
$$

This completes the proof.

\section{Manipulation of proof trees}

Fact 2.24 Given $j d g^{\prime}=C^{\prime}, A^{\prime} \vdash E[e]: \sigma^{\prime} \& b^{\prime}$; then there exists (at least one) judgement $j d g$ of form $C, A \vdash e: \sigma \& b$ such that $j d g$ occurs at $E$ in the inference tree for $j d g^{\prime}$.

Proof The proof is by induction in the inference tree for $j d g^{\prime}$. If $E=$ [] we can use $j d g=j d g^{\prime}$, so assume $E \neq[]$. Hence the last rule applied in the inference tree for $j d g^{\prime}$ is none of the following: (con), (id), (abs), or (rec). If (sub), (ins) or (gen) has been applied the induction hypothesis clearly yields the claim. So we are left with (app), (let) and (if); we only consider (app) as the other cases are similar. Then $E$ takes either the form $E_{1} e_{2}$ or the form $w_{1} E_{2}$; we consider the former only as the latter is similar.

The situation thus is that $E[e]=E_{1}[e] e_{2}$ so the left premise of $j d g^{\prime}$ is of form $C^{\prime \prime}, A^{\prime \prime} \vdash E_{1}[e]: \sigma^{\prime \prime} \& b^{\prime \prime}$ (abbreviated $j d g^{\prime \prime}$ ). Inductively we can assume that there exists $j d g$ which occurs at $E_{1}$ in the inference tree for $j d g^{\prime \prime}$; but this shows that $j d g$ occurs at $E$ in the inference tree for $j d g^{\prime}$.

Lemma 2.28 Suppose the judgement $j d g=(C, A \vdash e: \sigma \& b)$ occurs at $E$ with depth $n$ in the inference tree of $j d g^{\prime}=\left(C^{\prime}, A \vdash E[e]: \sigma^{\prime} \& b^{\prime}\right)$ where $C^{\prime}$ (and hence also $C$ ) is well-formed.

Let $c h$ be a channel identifier not in $E[e]$, and let $t$ be a type and $e_{0}$ an expression such that

$$
C \vdash\{t \mathrm{CHAN}\} \subseteq b \text { and } C, A[c h: t \text { chan }] \vdash e_{0}: \sigma \& b
$$


Then it also holds that

$$
C^{\prime} \vdash\{t \mathrm{CHAN}\} \subseteq b^{\prime} \text { and } C^{\prime}, A[c h: t \text { chan }] \vdash E\left[e_{0}\right]: \sigma^{\prime} \& b^{\prime} .
$$

Proof We perform induction in $n$ : if $n=0$ then $E=[], C^{\prime}=C, \sigma^{\prime}=\sigma, b^{\prime}=b$ and the claim is trivial.

If $n>1$ then by Fact 2.25 there exists judgement $j d g^{\prime \prime}=C^{\prime \prime}, A^{\prime \prime} \vdash e^{\prime \prime}: \sigma^{\prime \prime} \& b^{\prime \prime}$ and evaluation contexts $E_{1}$ and $E_{2}$ such that $E=E_{2}\left[E_{1}\right]$ and such that

$j d g$ occurs at $E_{1}$ with depth $<n$ in the inference tree for $j d g^{\prime \prime}$; and $j d g^{\prime \prime}$ occurs at $E_{2}$ with depth $<n$ in the inference tree for $j d g^{\prime}$.

So if $C \vdash\{t \mathrm{CHAN}\} \subseteq b$ and $C, A[c h: t$ chan $] \vdash e_{0}: \sigma \& b$ we can apply the induction hypothesis (with $j d g$ and $j d g^{\prime \prime}$ ) to infer that $C^{\prime \prime} \vdash\{t \mathrm{CHAN}\} \subseteq b^{\prime \prime}$ and that $C^{\prime \prime}, A[c h: t$ chan $] \vdash E_{1}\left[e_{0}\right]: \sigma^{\prime \prime} \& b^{\prime \prime}$; and by applying the induction hypothesis once more (with $j d g^{\prime \prime}$ and $j d g^{\prime}$ ) we can infer $C^{\prime} \vdash\{t \mathrm{CHAN}\} \subseteq b^{\prime}$ and $C^{\prime}, A[c h: t$ chan $] \vdash E_{2}\left[E_{1}\left[e_{0}\right]\right]: \sigma^{\prime} \& b^{\prime}$ which is as desired (due to Fact 2.14).

So we are left with the case $n=1$. We perform case analysis on $E$ :

$\underline{E=E_{1} e_{2}}:$ Here $E_{1}=[]$ and the situation is:

$$
\frac{j d g=C_{1}, A \vdash e_{1}:\left(t_{2} \rightarrow^{b} t_{1}\right) \& b_{1} \quad C_{2}, A \vdash e_{2}: t_{2} \& b_{2}}{j d g^{\prime}=\left(C_{1} \cup C_{2}\right), A \vdash e_{1} e_{2}: t_{1} \&\left(b_{1} \cup b_{2} \cup b\right)}
$$

and our assumptions are

$$
C_{1} \vdash\{t \mathrm{CHAN}\} \subseteq b_{1} \text { and } C_{1}, A[c h: t \text { chan }] \vdash e_{0}: t_{2} \rightarrow^{b} t_{1} \& b_{1}
$$

and we must show that

$$
\begin{aligned}
& C_{1} \cup C_{2} \vdash\{t \text { CHAN }\} \subseteq b_{1} \cup b_{2} \cup b \text { and } \\
& \left(C_{1} \cup C_{2}\right), A[c h: t \text { chan }] \vdash e_{0} e_{2}: t_{1} \&\left(b_{1} \cup b_{2} \cup b\right) .
\end{aligned}
$$

The former is a trivial consequence of the assumptions, and the latter will follow provided we can show that $C_{2}, A[c h: t$ chan $] \vdash e_{2}: t_{2} \& b_{2}$. But this follows from Fact 2.8 since $c h$ does not occur in $e_{2}$.

$\underline{E=w E_{2}}$ : Similar to the case above (now exploiting that for all $C$ it holds that $\left.C \vdash b_{2} \subseteq b_{1} \cup b_{2} \cup b\right)$. 
$\underline{E=\text { let } x=E_{1} \text { in } e_{2}:}$ Here $E_{1}=$ [] and the situation is:

$$
\frac{j d g=C_{1}, A \vdash e_{1}: t s_{1} \& b_{1} \quad C_{2}, A\left[x: t s_{1}\right] \vdash e_{2}: t_{2} \& b_{2}}{j d g^{\prime}=\left(C_{1} \cup C_{2}\right), A \vdash \operatorname{let} x=e_{1} \text { in } e_{2}: t_{2} \&\left(b_{1} \cup b_{2}\right)}
$$

and our assumptions are

$$
C_{1} \vdash\{t \mathrm{CHAN}\} \subseteq b_{1} \text { and } C_{1}, A[c h: t \text { chan }] \vdash e_{0}: t s_{1} \& b_{1}
$$

and we must show that

$$
\begin{aligned}
& C_{1} \cup C_{2} \vdash\{t \text { cHAN }\} \subseteq b_{1} \cup b_{2} \text { and } \\
& \left(C_{1} \cup C_{2}\right), A[c h: t \text { chan }] \vdash \text { let } x=e_{0} \text { in } e_{2}: t_{2} \&\left(b_{1} \cup b_{2}\right) .
\end{aligned}
$$

The former is a trivial consequence of the assumptions, and the latter will follow provided we can show that $C_{2}, A[c h: t$ chan $]\left[x: t s_{1}\right] \vdash e_{2}: t_{2} \& b_{2}$. But this follows from Fact 2.8 and Fact 2.7 since $c h \neq x$ and $c h$ does not occur in $e_{2}$.

$\underline{E=\text { if } E_{0} \text { then } e_{1} \text { else } e_{2}:}$ Here $E_{0}=$ [] and the situation is:

$$
\frac{j d g=C_{0}, A \vdash e_{0}: \text { bool \& } b_{0} \quad C_{1}, A \vdash e_{1}: t_{1} \& b_{1} \quad C_{2}, A \vdash e_{2}: t_{1} \& b_{2}}{j d g^{\prime}=\left(C_{0} \cup C_{1} \cup C_{2}\right), A \vdash \text { if } e_{0} \text { then } e_{1} \text { else } e_{2}: t_{1} \&\left(b_{0} \cup b_{1} \cup b_{2}\right)}
$$

and our assumptions are

$$
C_{0} \vdash\{t \mathrm{CHAN}\} \subseteq b_{0} \text { and } C_{0}, A[c h: t \text { chan }] \vdash e_{0}: \text { bool } \& b_{0}
$$

and we must show that

$$
\begin{aligned}
& C_{0} \cup C_{1} \cup C_{2} \vdash\{t \mathrm{CHAN}\} \subseteq b_{0} \cup b_{1} \cup b_{2} \text { and } \\
& \left(C_{0} \cup C_{1} \cup C_{2}\right), A[c h: t \text { chan }] \vdash \text { if } e_{0} \text { then } e_{1} \text { else } e_{2}: t_{1} \&\left(b_{0} \cup b_{1} \cup b_{2}\right) .
\end{aligned}
$$

The former is a trivial consequence of the assumptions, and the latter will follow if we can show $C_{1}, A[c h: t$ chan $] \vdash e_{1}: t_{1} \& b_{1}$ and $C_{2}, A[c h: t$ chan $] \vdash e_{2}: t_{1} \& b_{2}$. But this follows from Fact 2.8 since $c h$ does not occur in $e_{1}$ or $e_{2}$.

$\underline{E=[]:}$ In this case $j d g^{\prime}$ follows from $j d g$ by one application of either (sub),

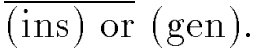


(sub) has been applied: the situation is

$$
\frac{j d g=C, A \vdash e: t_{0} \& b}{j d g^{\prime}=C, A \vdash e: t^{\prime} \& b^{\prime}}
$$

where $C \vdash t_{0} \subseteq t^{\prime}$ and $C \vdash b \subseteq b^{\prime}$. Our assumptions are

$$
C \vdash\{t \mathrm{CHAN}\} \subseteq b \text { and } C, A[c h: t \text { chan }] \vdash e_{0}: t_{0} \& b
$$

and we must show that

$$
C \vdash\{t \mathrm{CHAN}\} \subseteq b^{\prime} \text { and } C, A[c h: t \text { chan }] \vdash e_{0}: t^{\prime} \& b^{\prime} .
$$

But this is trivial.

(ins) has been applied: the situation is

$$
\frac{j d g=C, A \vdash e: \forall\left(\vec{\alpha} \vec{\beta}: C_{0}\right) \cdot t_{0} \& b}{j d g^{\prime}=C, A \vdash e: S_{0} t_{0} \& b}
$$

where $\forall\left(\vec{\alpha} \vec{\beta}: C_{0}\right) . t_{0}$ is solvable from $C$ by $S_{0}$. Our assumptions are

$$
C \vdash\{t \mathrm{CHAN}\} \subseteq b \text { and } C, A[c h: t \text { chan }] \vdash e_{0}: \forall\left(\vec{\alpha} \vec{\beta}: C_{0}\right) . t_{0} \& b
$$

and we must show that

$$
C \vdash\{t \mathrm{CHAN}\} \subseteq b \text { and } C, A[c h: t \text { chan }] \vdash e_{0}: S_{0} t_{0} \& b
$$

But this is trivial.

(gen) has been applied: this is the really interesting case! The situation is

$$
\frac{j d g=C \cup C_{0}, A \vdash e: t_{0} \& b}{j d g^{\prime}=C, A \vdash e: \forall\left(\vec{\alpha} \vec{\beta}: C_{0}\right) . t_{0} \& b}
$$

where $\forall\left(\vec{\alpha} \vec{\beta}: C_{0}\right) . t_{0}$ is well-formed and where $\{\vec{\alpha} \vec{\beta}\} \cap F V(C, A, b)=\emptyset$ and where there exists $S$ with $\operatorname{Dom}(S) \subseteq\{\vec{\alpha} \vec{\beta}\}$ such that $C \vdash S C_{0}$. Our assumptions are

$$
\begin{aligned}
& C \cup C_{0} \vdash\{t \text { CHAN }\} \subseteq b \\
& C \cup C_{0}, A[c h: t \text { chan }] \vdash e_{0}: t_{0} \& b
\end{aligned}
$$

and we must show that 


$$
\begin{aligned}
& C \vdash\{t \text { CHAN }\} \subseteq b \\
& C, A[\text { ch }: t \text { chan }] \vdash e_{0}: \forall\left(\vec{\alpha} \vec{\beta}: C_{0}\right) . t_{0} \& b
\end{aligned}
$$

It will suffice to prove

$$
\{\vec{\alpha} \vec{\beta}\} \cap F V(t)=\emptyset
$$

for then (1) and Lemma 2.5(a) give that $C \cup S C_{0} \vdash t$ CHAN $\subseteq b$ which (by Lemma 2.6) implies (3); and we will be able to use (gen) to arrive at (4) from (2).

So we are left with the task of proving (5). Since $\forall\left(\vec{\alpha} \vec{\beta}: C_{0}\right)$. $t_{0}$ is well-formed it holds that $\{\vec{\alpha} \vec{\beta}\}^{C_{0} \uparrow}=\{\vec{\alpha} \vec{\beta}\}$, that is

$$
\text { if } C_{0} \vdash \gamma \leftarrow \gamma^{\prime} \text { with } \gamma \in\{\vec{\alpha} \vec{\beta}\} \text { then also } \gamma^{\prime} \in\{\vec{\alpha} \vec{\beta}\} \text {. }
$$

By Fact 2.2 we are able to infer (as $\{\vec{\alpha} \vec{\beta}\} \cap F V(C)=\emptyset$ ) that

$$
\text { if } C \cup C_{0} \vdash \gamma \leftarrow \gamma^{\prime} \text { with } \gamma \in\{\vec{\alpha} \vec{\beta}\} \text { then } C_{0} \vdash \gamma \leftarrow \gamma^{\prime} \text {. }
$$

By combining (6) and (7) we infer that

$$
\text { if } C \cup C_{0} \vdash \gamma \leftarrow \gamma^{\prime} \text { with } \gamma \in\{\vec{\alpha} \vec{\beta}\} \text { then also } \gamma^{\prime} \in\{\vec{\alpha} \vec{\beta}\}
$$

which amounts to $\{\vec{\alpha} \vec{\beta}\}^{\left(C \cup C_{0}\right) \uparrow}=\{\vec{\alpha} \vec{\beta}\}$. Since $\{\vec{\alpha} \vec{\beta}\} \cap F V(b)=\emptyset$ we have $\{\vec{\alpha} \vec{\beta}\}^{\left(C \cup C_{0}\right) \uparrow} \cap F V(b)=\emptyset$, and hence we do not have $C \cup C_{0} \vdash \gamma \leftarrow^{*} \gamma^{\prime}$ for any $\gamma \in\{\vec{\alpha} \vec{\beta}\}$ and $\gamma^{\prime} \in F V(b)$. But this is just another way of saying that

$$
\{\vec{\alpha} \vec{\beta}\} \cap F V(b)^{\left(C \cup C_{0}\right) \downarrow}=\emptyset .
$$

From (1) and from Lemma 2.4 (which can be applied since we know that $C \cup C_{0}$ is well-formed) we infer that

$$
F V(t)^{\left(C \cup C_{0}\right) \downarrow} \subseteq F V(b)^{\left(C \cup C_{0}\right) \downarrow} .
$$

Combining (8) and (9) we get $\{\vec{\alpha} \vec{\beta}\} \cap F V(t)^{\left(C \cup C_{0}\right) \downarrow}=\emptyset$ which trivially implies (5). This completes the proof.

Lemma 2.29 Suppose the judgement $j d g=C, A \vdash e: \sigma \& b$ occurs at $E$ with depth $n$ in the constraint-saturated inference tree of $j d g^{\prime}=C^{\prime}, A \vdash_{c} e^{\prime}: \sigma^{\prime} \& b^{\prime}$ where $C^{\prime}$ (and hence also $C$ ) is well-formed. 
Then $C^{\prime} \subseteq C$, and there exists $S$ with $\operatorname{Dom}(S) \cap F V(A)=\emptyset$ such that $C^{\prime} \vdash S C$.

Proof We perform induction in $n$ : if $n=0$ then $C^{\prime}=C$ and we can use $S=\mathrm{Id}$.

If $n>1$ then by Fact 2.25 there exists judgement $j d g^{\prime \prime}=C^{\prime \prime}, A^{\prime \prime} \vdash e^{\prime \prime}: \sigma^{\prime \prime} \& b^{\prime \prime}$ and evaluation contexts $E_{1}$ and $E_{2}$ such that

$j d g$ occurs at $E_{1}$ with depth $<n$ in the inference tree for $j d g^{\prime \prime}$; and $j d g^{\prime \prime}$ occurs at $E_{2}$ with depth $<n$ in the inference tree for $j d g^{\prime}$.

We can thus apply the induction hypothesis twice to infer that $C^{\prime} \subseteq C^{\prime \prime} \subseteq C$ and that there exists $S_{1}, S_{2}$ with $\operatorname{Dom}\left(S_{1}\right) \cap F V(A)=\emptyset=\operatorname{Dom}\left(S_{2}\right) \cap F V(A)$ such that $C^{\prime} \vdash S_{2} C^{\prime \prime}$ and $C^{\prime \prime} \vdash S_{1} C$. But then we by Lemma 2.5 and 2.6 arrive at $C^{\prime} \vdash S_{2} S_{1} C$, where clearly $\operatorname{Dom}\left(S_{2} S_{1}\right) \cap F V(A)=\emptyset$.

So we are left with the case $n=1$. We perform case analysis on the inference rule applied. The only interesting case is (gen), for otherwise we have $C^{\prime}=C$ due to our assumption about the inference tree being constraint saturated and hence we can use $S=\mathrm{Id}$. The situation thus is

$$
\frac{j d g=C \cup C_{0}, A \vdash e: t_{0} \& b}{j d g^{\prime}=C, A \vdash e: \forall\left(\vec{\alpha} \vec{\beta}: C_{0}\right) \cdot t_{0} \& b}
$$

where $\{\vec{\alpha} \vec{\beta}\} \cap F V(C, A, b)=\emptyset$ and where there exists $S$ with $\operatorname{Dom}(S) \subseteq\{\vec{\alpha} \vec{\beta}\}$ such that $C \vdash S C_{0}$. Our task can be accomplished by showing that $C \subseteq C \cup C_{0}$ and that $\operatorname{Dom}(S) \cap F V(A)=\emptyset$ and that $C \vdash S C_{0}$ and that $C \vdash S C$. But all this follows directly.

Lemma 2.30 Suppose that $C$ is well-formed and that

$$
C, A \vdash_{s} c e_{1} \cdots e_{n}: t \& b(n \geq 0)
$$

and that TypeOf $(c)$ is of form

$$
\forall\left(\vec{\alpha} \vec{\beta}: C_{0}\right) \cdot t_{1}^{\prime} \rightarrow_{1}^{b_{1}^{\prime}} \cdots t_{m}^{\prime} \rightarrow_{m}^{b_{m}^{\prime}} t^{\prime \prime}
$$

where we demand that if $c$ is a base function then $m \geq n$. Then in all cases (i.e. also if $c$ is a constructor) we can write

$$
\text { TypeOf }(c)=\forall\left(\vec{\alpha} \vec{\beta}: C_{0}\right) \cdot t_{1}^{\prime} \rightarrow_{1}^{b_{1}^{\prime}} \cdots t_{n}^{\prime} \rightarrow_{n}^{b_{n}^{\prime}} \quad t^{\prime}
$$

and there exists $S, t_{1} \cdots t_{n}$, and $b_{1} \cdots b_{n}$, such that 
$\operatorname{Dom}(S) \subseteq\{\vec{\alpha} \vec{\beta}\}$ and $C \vdash S C_{0}$ and $C \vdash S t^{\prime} \subseteq t ;$

for all $i \in\{1 \cdots n\}: C, A \vdash_{s} e_{i}: t_{i} \& b_{i}$ and $C \vdash t_{i} \subseteq S t_{i}^{\prime}$ and $C \vdash b_{i} \subseteq b$ and $C \vdash S b_{i}^{\prime} \subseteq b$.

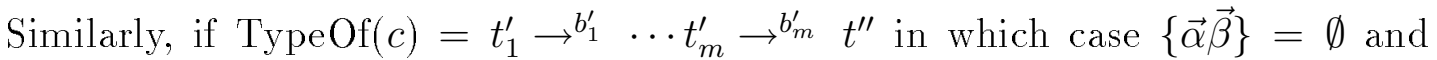
$C_{0}=\emptyset$ (so we have $S=\mathrm{Id}$ ).

Proof We perform induction in $n$. If $n=0$ we can trivially always assume (10), i.e. that TypeOf $(c)$ takes the form $\forall\left(\vec{\alpha} \vec{\beta}: C_{0}\right)$. $t^{\prime}$, and the claim is that if $C, A \vdash_{s} c: t \& b$ then there exists $S$ with $\operatorname{Dom}(S) \subseteq\{\vec{\alpha} \vec{\beta}\}$ and $C \vdash S C_{0}$ such that $C \vdash S t^{\prime} \subseteq t$. But since $C, A \vdash_{s} c: t \& b$ is constructed by an application of (con) followed by an application of (ins) followed by an application of (sub), this is immediate.

Next consider the inductive step. The situation is that there exists $t_{n}, t^{-}, b_{n}, b^{\prime}$ and $b^{\prime \prime}$ such that

$$
\frac{C, A \vdash_{s} c e_{1} \cdots e_{n-1}: t_{n} \rightarrow^{b^{\prime \prime}} t^{-} \& b^{\prime} \quad C, A \vdash_{s} e_{n}: t_{n} \& b_{n}}{C, A \vdash_{s} c e_{1} \cdots e_{n}: t \& b}(\operatorname{app})(\mathrm{sub})
$$

where $C \vdash t^{-} \subseteq t$ and $C \vdash b^{\prime} \cup b_{n} \cup b^{\prime \prime} \subseteq b$.

By the induction hypothesis we infer that in all cases it holds that

TypeOf(c) takes the form $\forall\left(\vec{\alpha} \vec{\beta}: C_{0}\right) . t_{1}^{\prime} \rightarrow_{1}^{b_{1}^{\prime}} \cdots t_{n-1}^{\prime} \rightarrow_{n-1}^{b_{n-1}^{\prime}} t^{\prime \prime \prime}$ and that there exists $S, t_{1} \cdots t_{n-1}$, and $b_{1} \cdots b_{n-1}$, such that

$\operatorname{Dom}(S) \subseteq\{\vec{\alpha} \vec{\beta}\}$ and $C \vdash S C_{0}$;

$C \vdash S t^{\prime \prime \prime} \subseteq t_{n} \rightarrow^{b^{\prime \prime}} t^{-}$

for all $i \in\{1 \cdots n-1\}: C, A \vdash_{s} e_{i}: t_{i} \& b_{i}$ and $C \vdash t_{i} \subseteq S t_{i}^{\prime}$ and $C \vdash b_{i} \subseteq b^{\prime} \subseteq b$ and $C \vdash S b_{i}^{\prime} \subseteq b^{\prime} \subseteq b$.

Since $C$ is well-formed we can apply Lemma 2.3 on (11) to infer that $S t^{\prime \prime \prime}$ is a function type. If $c$ is a constructor Fact 2.1 tells us that $t^{\prime \prime \prime}$ cannot be a variable; hence in all cases we can write $t^{\prime \prime \prime}=t_{n}^{\prime} \rightarrow_{n}^{b_{n}^{\prime}} t^{\prime}$ which amounts to (10). Lemma 2.3 further tells us that $C \vdash t_{n} \subseteq S t_{n}^{\prime}$ and that $C \vdash S b_{n}^{\prime} \subseteq b^{\prime \prime} \subseteq b$ and that $C \vdash S t^{\prime} \subseteq t^{-} \subseteq t$. Thus all our proof obligations are fulfilled.

Lemma 2.31 Suppose that $C, A\left[x: \sigma^{\prime}\right] \vdash e: \sigma \& b$ and that $C, A \vdash e^{\prime}: \sigma^{\prime} \& \emptyset$; then $C, A \vdash e\left[e^{\prime} / x\right]: \sigma \& b$.

Proof Induction in the shape of the proof tree for $C, A\left[x: \sigma^{\prime}\right] \vdash e: \sigma \& b$ which we by Fact 2.9 can assume to be constraint saturated. We perform case analysis on the last rule applied: 
(con) has been applied: Then $e$ is a constant, and $e\left[e^{\prime} / x\right]=e$ so the claim is clear.

(id) has been applied: Then $e$ is an identifier $y$. If $y \neq x$ then $e\left[e^{\prime} / x\right]=e$ and the claim is clear since $A\left[x: \sigma^{\prime}\right](y)=A(y)$.

If $y=x$ then $\sigma=\sigma^{\prime}$ and $b=\emptyset$. Since $e\left[e^{\prime} / x\right]=e^{\prime}$ the claim follows from the second part of the assumption.

(abs) has been applied: Here the inference takes the form

$$
\frac{C, A\left[x: \sigma^{\prime}\right]\left[y: t_{1}\right] \vdash e: t_{2} \& b}{C, A\left[x: \sigma^{\prime}\right] \vdash \text { fn } y \Rightarrow e: t_{1} \rightarrow^{b} t_{2} \& \emptyset}
$$

where we can assume (by suitable alpha-renaming) that $y \neq x$ and that $y$ does not occur in $e^{\prime}$. Hence we can apply Fact 2.7 and Fact 2.8 to get

$C, A\left[y: t_{1}\right]\left[x: \sigma^{\prime}\right] \vdash e: t_{2} \& b$ with the same shape as the premise and $C, A\left[y: t_{1}\right] \vdash e^{\prime}: \sigma^{\prime} \& \emptyset$.

We can thus apply the induction hypothesis and subsequently use (abs) to construct an inference tree whose last inference is

$$
\frac{C, A\left[y: t_{1}\right] \vdash e\left[e^{\prime} / x\right]: t_{2} \& b}{C, A \vdash \operatorname{fn} y \Rightarrow e\left[e^{\prime} / x\right]: t_{1} \rightarrow^{b} t_{2} \& \emptyset}
$$

which is as desired since $($ fn $y \Rightarrow e)\left[e^{\prime} / x\right]=\left(\right.$ fn $\left.y \Rightarrow e\left[e^{\prime} / x\right]\right)$.

(app) has been applied: Here the inference (which was assumed to be constraint saturated) takes the form

$$
\frac{C, A\left[x: \sigma^{\prime}\right] \vdash e_{1}: t_{2} \rightarrow^{b} t_{1} \& b_{1} \quad C, A\left[x: \sigma^{\prime}\right] \vdash e_{2}: t_{2} \& b_{2}}{C, A\left[x: \sigma^{\prime}\right] \vdash e_{1} e_{2}: t_{1} \&\left(b_{1} \cup b_{2} \cup b\right)}
$$

where we can apply the induction hypothesis twice and subsequently use (app) to construct an inference tree whose last inference is

$$
\frac{C, A \vdash e_{1}\left[e^{\prime} / x\right]: t_{2} \rightarrow^{b} t_{1} \& b_{1} \quad C, A \vdash e_{2}\left[e^{\prime} / x\right]: t_{2} \& b_{2}}{C, A \vdash e_{1}\left[e^{\prime} / x\right] e_{2}\left[e^{\prime} / x\right]: t_{1} \&\left(b_{1} \cup b_{2} \cup b\right)}
$$

which is as desired since $\left(e_{1} e_{2}\right)\left[e^{\prime} / x\right]=e_{1}\left[e^{\prime} / x\right] e_{2}\left[e^{\prime} / x\right]$. 
(let), (rec) or (if) has been applied: Similar to the above two cases, exploiting Fact 2.7 and Fact 2.8 and we only spell the case (rec) out in detail. Here the inference takes the form

$$
\frac{C, A\left[x: \sigma^{\prime}\right][f: t] \vdash \text { fn } y \Rightarrow e: t \& b}{C, A\left[x: \sigma^{\prime}\right] \vdash \operatorname{rec} f y \Rightarrow e: t \& b}
$$

where we can assume that $y \neq x, f \neq x$ and that neither $y$ nor $f$ occurs in $e^{\prime}$. Hence we can apply Fact 2.7 and Fact 2.8 to get

$C, A[f: t]\left[x: \sigma^{\prime}\right] \vdash$ fn $y \Rightarrow e: t \& b$ with the same shape as the premise and $C, A[f: t] \vdash e^{\prime}: \sigma^{\prime} \& \emptyset$.

We can thus apply the induction hypothesis to infer

$$
C, A[f: t] \vdash(\text { fn } y \Rightarrow e)\left[e^{\prime} / x\right]: t \& b
$$

which since $y \neq x$ and $y$ is not free in $e^{\prime}$ amounts to

$$
C, A[f: t] \vdash \text { fn } y \Rightarrow e\left[e^{\prime} / x\right]: t \& b .
$$

By applying (rec) we get

$$
C, A \vdash \operatorname{rec} f y \Rightarrow e\left[e^{\prime} / x\right]: t \& b
$$

which is as desired since $(\operatorname{rec} f y \Rightarrow e)\left[e^{\prime} / x\right]=\left(\operatorname{rec} f y \Rightarrow e\left[e^{\prime} / x\right]\right)$.

(sub) has been applied: Here the inference takes the form

$$
\frac{C, A\left[x: \sigma^{\prime}\right] \vdash e: t \& b}{C, A\left[x: \sigma^{\prime}\right] \vdash e: t^{\prime} \& b^{\prime}} \quad \text { with } C \vdash t \subseteq t^{\prime} \text { and } C \vdash b \subseteq b^{\prime}
$$

so we can apply the induction hypothesis and subsequently use (sub) to construct an inference tree whose last inference is

$$
\frac{C, A \vdash e\left[e^{\prime} / x\right]: t \& b}{C, A \vdash e\left[e^{\prime} / x\right]: t^{\prime} \& b^{\prime}}
$$

(ins) has been applied: Similar to the above case. 
(gen) has been applied: Here the inference takes the form

$$
\frac{C \cup C_{0}, A\left[x: \sigma^{\prime}\right] \vdash e: t_{0} \& b}{C, A\left[x: \sigma^{\prime}\right] \vdash e: t s \& b}
$$

where $t s=\forall\left(\vec{\alpha} \vec{\beta}: C_{0}\right) \cdot t_{0}$ is well-formed, solvable from $C$, and satisfies $\{\vec{\alpha} \vec{\beta}\} \cap$ $F V\left(C, A\left[x: \sigma^{\prime}\right], b\right)=\emptyset$. By Lemma 2.6 we have

$$
C \cup C_{0}, A \vdash e^{\prime}: \sigma^{\prime} \& \emptyset
$$

so we can apply the induction hypothesis to get

$$
C \cup C_{0}, A \vdash e\left[e^{\prime} / x\right]: t_{0} \& b
$$

We can then apply (gen) (since $\{\vec{\alpha} \vec{\beta}\} \cap F V(C, A, b)=\emptyset$ ) to arrive at the desired judgement $C, A \vdash e\left[e^{\prime} / x\right]: t s \& b$.

Lemma 2.32 Suppose that $C, A \vdash_{s} w: \sigma \& b$ with $C$ well-formed;

then $C, A \vdash_{s} w: \sigma \& \emptyset$ and with the same shape.

Proof It is enough to consider the case where $\sigma$ is a type $t$, for if the inference

$$
\frac{C \cup C_{0}, A \vdash w: t_{0} \& b}{C, A \vdash w: \forall\left(\vec{\alpha} \vec{\beta}: C_{0}\right) \cdot t_{0} \& b} \text { (gen) }
$$

is valid it remains valid when $b$ is replaced by $\emptyset$. We now prove the claim by induction in the size of $w$, and the only interesting case is where $w=c w_{1} \cdots w_{n}$ for $n \geq 1$ and with $c$ being a constructor.

Lemma 2.30 combined with Fact 2.1 tells us that

TypeOf(c) takes the form $\forall(\vec{\alpha} \vec{\beta}: \emptyset) . t_{1}^{\prime} \rightarrow^{\emptyset} \cdots t_{n}^{\prime} \rightarrow^{\emptyset} t^{\prime}$

and Lemma 2.30 further tells us that there exists $t_{1} \cdots t_{n}, b_{1} \cdots b_{n}$, and $S$ with $\operatorname{Dom}(S) \subseteq\{\vec{\alpha} \vec{\beta}\}$ such that

$$
\begin{aligned}
& C \vdash S t^{\prime} \subseteq t \\
& \text { for all } i \in\{1 \cdots n\}: C, A \vdash_{s} w_{i}: t_{i} \& b_{i} \text { and } C \vdash t_{i} \subseteq S t_{i}^{\prime} .
\end{aligned}
$$

The induction hypothesis tells us that

$$
\text { for all } i \in\{1 \cdots n\}: C, A \vdash_{s} w_{i}: t_{i} \& \emptyset
$$

and by using (con), (ins) and (sub) we have 


$$
C, A \vdash_{s} c: t_{1} \rightarrow^{\emptyset} \cdots t_{n} \rightarrow^{\emptyset} t \& \emptyset
$$

This shows that we can construct the desired judgement $C, A \vdash_{s} \quad c w_{1} \cdots w_{n}: t \& \emptyset$ (where the applications of (sub) are justified by $C \vdash \emptyset \cup \emptyset \cup \emptyset \subseteq \emptyset$ ). 